\title{
A BOOLEAN ALGEBRA WITH FEW SUBALGEBRAS, INTERVAL BOOLEAN ALGEBRAS AND RETRACTIVENESS
}

BY

\author{
MATATYAHU RUBIN
}

\begin{abstract}
Using $\nabla_{\boldsymbol{N}_{1}}$ we construct a Boolean algebra $B$ of power $\boldsymbol{\kappa}_{1}$, with the following properties: (a) $B$ has just $\kappa_{1}$ subalgebras. (b) Every uncountable subset of $B$ contains a countable independent set, a chain of order type $\eta$, and three distinct elements $a, b$ and $c$, such that $a \cap b=c$. (a) refutes a conjecture of J. D. Monk, (b) answers a question of R. McKenzie. $B$ is embeddable in $P(\omega)$. A variant of the construction yields an almost Jonson Boolean algebra. We prove that every subalgebra of an interval algebra is retractive. This answers affirmatively a conjecture of $B$. Rotman. Assuming MA or the existence of a Suslin tree we find a retractive BA not embeddable in an interval algebra. This refutes a conjecture of B. Rotman. We prove that an uncountable subalgebra of an interval algebra contains an uncountable chain or an uncountable antichain. Assuming $\mathrm{CH}$ we prove that the theory of Boolean algebras in Magidor's and Malitz's language is undecidable. This answers a question of $\mathrm{M}$. Weese.
\end{abstract}

1. Introduction. In this paper we describe a construction of Boolean algebras (BA's). Our construction yields counterexamples to several questions about BA's. However, we use $\diamond_{N_{1}}$, so most of the questions remain open in ZFC $+\mathrm{CH}$.

We first construct a BA $B$ of power $\boldsymbol{\aleph}_{1}$ with the following properties: (1) $B$ has just $\boldsymbol{}_{1}$ subalgebras. (2) Every uncountable subset of $B$ contains: a chain of the order type of the rationals, an infinite independent set, and three distinct elements $a, b, c$ such that $a \cap b=c$. (3) $B$ is retractive but is not embeddable in an interval algebra. (See Definitions 1.1 and 1.2.)

Property (1) refutes a conjecture of J. D. Monk, that an infinite BA $A$ has always $2^{|A|}$ subalgebras. In fact, Shelah proved that $B$ has just $\aleph_{1}$ lower or upper subsemilattices.

$\mathrm{R}$. McKenzie [Mc] proved that Monk's conjecture is true if $|A|$ is a strong limit. Let us survey his proof. A subset $P$ of an algebra $M$ is called irredundant, if no element $a \in P$ belongs to the subalgebra generated by $P-\{a\}$. Clearly, distinct subsets of an irredundant set generate distinct subalgebras; so if $P \subseteq M$ is irredundant, then $M$ has at least $2^{|P|}$ subalgebras. McKenzie then proved that the subalgebra generated by a maximal irredundant subset of a BA $B$ is dense in $B$; that is every nonzero element of $B$ is greater than some nonzero element of that subalgebra. Clearly, by Zorn's lemma, every algebra contains a maximal irredundant subset. So

Received by the editors November 28, 1977 and, in revised form, October 11, 1981

1980 Mathematics Subject Classification. Primary 06E05; Secondary 03E35, 06A05.

'RemarK. S. Koppelberg proved that any uncountable subset of an interval algebra contains an uncountable irredundant subset. This is still another way to prove 5.7.

(C) 1983 American Mathematical Society 0002-9947/82/0000-0162/\$07.75 
if $B$ is a $\mathrm{BA}$, and $|B|$ is a strong limit, then $B$ contains a maximal irredundant set $P$, and $|P|$ has to be equal to $|B|$. So $B$ has $2^{|B|}$ subalgebras.

McKenzie then asked whether every infinite BA contains an irredundant set of the same cardinality.

Property (2) refutes this in a strong way. In fact, the detailed formulation of (2) (Theorem 4.6) is the strongest possible in this direction. That is, we divide the countable and finite configurations of subsets of a BA into two classes: $\mathcal{L}_{1}$ and $\mathcal{L}_{2}$. Every configuration in $\mathcal{L}_{1}$ appears as a subset of every uncountable subset of our $\mathrm{BA}$; on the other hand if $B$ is an uncountable BA, there is always an uncountable subset $P$ of $B$, such that no configuration in $\mathcal{L}_{2}$ is realized by a subset of $P$. (This last fact is trivial, and depends on ZFC.) For example, the configuration: " $a \neq 0 \neq b$ and $a \cap b=0$ " is in $\mathcal{L}_{2}$. The configuration: " $\left\{b_{i} \mid i \in \omega\right\} ; i \neq j \Rightarrow b_{i} \neq b_{j} ; b_{0} \neq 0$; and $0<j<i \Rightarrow b_{0}=b_{i} \cap b_{j}$ and $1 \neq b_{i} \cup b_{j}$ " is in $e_{1}$.

Definition 1.1. A BA $C$ is retractive, if for every ideal $I$ in $C$, there is a subalgebra $C^{\prime}$ of $C$, such that for every $b \in C,\left|b / I \cap C^{\prime}\right|=1$.

DeFINITION 1.2. Let $\langle I,\langle\rangle$ be a linear ordering. The interval algebra based on $I$, $B(I)$, is the subalgebra of the power set of $I$ generated by the set $\left\{V_{a} \mid a \in I\right\}$, where $V_{a}=\{x \mid x \in I$ and $x \leqslant a\}$. An interval algebra is a BA which is isomorphic to $B(I)$ for some linear ordering $\langle I,<\rangle$.

B. Rotman [R, Conjecture A] conjectured that every retractive BA is embeddable in an interval algebra. By property (3) our BA is a strong counterexample to this conjecture, because every subalgebra of our BA which is embeddable in an interval algebra, is of power $\leqslant \boldsymbol{\aleph}_{0}$. However in $\S 6$ we find simpler but weaker counterexamples, assuming either MA or the existence of a Suslin tree.

Definition 1.3. A subset $P$ of a partial ordering is called a chain if every two elements of $P$ are comparable. $P$ is called an antichain if every two distinct elements of $P$ are incomparable. Note that this differs from the more common definition of an antichain. We prove that if $B$ is a subalgebra of an interval algebra, and $|B|$ is regular, then $B$ contains a chain or an antichain of power $|B|$. By property (2), the BA $B$ we construct does not contain uncountable chains or antichains, so it is not embeddable in an interval algebra. On the other hand if $I \subseteq B$ is a dense ideal, then $B / I$ is countable; this property implies retractiveness.

Partial orderings without chains and antichains have been studied extensively. An account of what was done and some open questions can be found in [DMR]. The following question which we did not succeed in solving does not appear in [DMR].

Question. Does $\mathrm{MA}+\left({ }_{\neg} \mathrm{CH}\right)$ imply that every uncountable BA contains an uncountable chain or an uncountable antichain?

We conjecture that the answer to the above question is negative.

Another question that seems to us interesting and not easy is the following.

Question. Does $(\mathrm{CH})$ imply that there is an uncountable BA $B$ such that every uncountable subset of $B$ contains a triple of distinct elements, $a, b, c$ such that $a \cap b=c$.

We conjecture that also the answer to this question is negative. 
Definition 1.4. Let $M$ be an algebra of power $\kappa . M$ is called almost Jónson (AJ), if for every subalgebra $N$ of $M$ of power $\kappa$, there is a subset $P$ of $M$ of power $<\kappa$, such that $N \cup P$ generates $M$.

An algebra $M$ has the $\mu$-intersection property, if the intersection of any two subalgebras of $M$ of power $\mu$ is of power $\mu$. ( $M$ has the $\mu$-IP.)

Notation. If $A$ is a BA let $I(A)=\left\{a||\{b \mid b \subseteq a\} \mid \leqslant \boldsymbol{\aleph}_{0}\right\}$.

By changing slightly the construction, we get a BA $B$ of power $\aleph_{1}$ with the following properties: (1) $B$ is an AJ, $\boldsymbol{\aleph}_{1}$-IP BA with just $\boldsymbol{\aleph}_{1}$ lower or upper subsemilattices. (2) $I(B)$ is an AJ lattice, and it is $\boldsymbol{\aleph}_{1}$-IP lower semilattice.

Notice that these are the best possible results of this kind; in particular, there is no Jónson BA.

In $\$ 5$ we show that the theory of BA's in Magidor and Malitz's language, $L^{2}$, is undecidable. We use the BA's constructed by Bonnet in [B1], thus we assume $\mathrm{CH}$.

This is an answer to a question of $\mathrm{M}$. Weese. Weese [W] proved that the theory of BA's in the language $L^{1}$ (where $Q^{1} x \varphi(x)$ means: there are uncountably many elements satisfying $\varphi$ ) is decidable.

Malitz asked whether there is a first order theory whose set of consequences in $L^{1}$ is decidable, but whose set of consequences in $L^{2}$ is undecidable. So assuming $\mathrm{CH}$ the theory of BA's is such an example; however we believe that $\mathrm{CH}$ is not needed and other examples must have been known before.

Let us mention what happens in higher cardinals. Using his omitting type theorem Shelah [S1] proved that if $\nabla_{\lambda^{-}}$and $\nabla_{\lambda^{+}}$hold, then there is a $\lambda$-saturated BA $B$ of power $\lambda^{+}$with the analogous properties. So in property (2) configurations of power $\lambda$ replace our countable configurations. However, Monk showed that a BA that contains an uncountable independent set is not retractive.

In $\S 6$ we make a modest contribution to the question: "When is the free product of two BA's retractive?" This question was raised by B. Rotman. Note that in [R] Rotman proved that the free product of an infinite BA and an uncountable BA is not embeddable in an interval algebra.

Our construction resembles Magidor and Malitz's proof of the compactness of $L^{<\omega}[\mathbf{M M}]$. Recently Shelah [S2] proved a theorem that generalizes our construction; however it does not imply the results presented here, but rather gives general conditions under which such constructions can be carried out.

I would like to thank F. Galvin, R. Laver, J. D. Monk, J. Mycielski, S. Shelah, Y. Gurevich, S. Theodorevich, and E. van Douwen for helpful discussions.

\section{Notations.}

Boolean algebras. A Boolean algebra (BA) is a structure of the form $\langle B, \cup, \cap$, -, 0,1$\rangle$. The letters $A$ and $B$ always denote BA's. $A, B$ denote both the Boolean algebra and its universe. $\subseteq$ denotes the partial ordering in a BA; $a \subsetneq b$ means $a \subseteq b$ and $a \neq b . a \triangle b$ denotes $(a-b) \cup(b-a)$. When we have to distinguish between the units of different BA's, we denote by $1_{B}, 0_{B}$ the 1 and 0 of $B$. If $a, b \in B$ and $a \cap b=0$, then $a$ and $b$ are said to be disjoint. 
$\operatorname{At}(B)$ denotes the set of atoms of $B$. If $P$ is a subset of $B$, then $\operatorname{cl}(P)$ is the subalgebra of $B$ generated by $P$. A subset $P$ of $B$ is dense in $B$, if for every $b \in B-\{0\}$, there is $c \in P, c \neq 0$, such that $c \subseteq b$.

If $C$ and $B$ are BA's, then $C \subseteq B$ always means that $C$ is a subalgebra of $B$. However, if $C$ is not a BA, then $C \subseteq B$ means that $C$ is a subset of the universe of $B$.

An ideal $I$ in a BA $B$ is a nonempty subset of $B$ that does not contain 1, is closed under $\cup$, and if $a \in I$ and $B \ni b \subseteq a$ then $b \in I$. If $I$ is an ideal in $B$, and $a \in B$ then $a / I=\{b \mid b \in B$ and $a \triangle b \in I\} . B / I=\{b / I \mid b \in B\} . B / I$ is regarded as a BA.

If $a \in B-\{0\}$, then $B \mid a$ is the BA induced by $B$ on the set $\{b \mid B \ni b \subseteq a\}$. If $I$ is an ideal in $B$, then $I \uparrow a=I \cap\{b \mid B \ni b \subseteq a\}$. If $a \notin I$, then $I \uparrow a$ is an ideal in $B \uparrow a$.

If $\varphi: B \rightarrow A$ is a homomorphism, then $\operatorname{ker}(\varphi)=\{a \mid a \in B$ and $\varphi(a)=0\} \cdot \operatorname{ker}(\varphi)$ is an ideal in $B$.

Partial orderings. If $\langle P,\langle\rangle$ is any partial ordering, $a, b \in P$, then $(a, b)=\{x \mid x$ $\in P$ and $a<x<b\},[a, b]=\{x \mid x \in P$ and $a \leqslant x \leqslant b\} .(a, b]$ and $[a, b)$ are defined similarly.

If $\langle P,\langle\rangle$ is a partial ordering $a \in P$ and $D \subseteq P$, then $a<D$ means that for every $d \in D a<d$. $D<a$ is defined similarly.

Sets and models. The cardinality of a set $D$ is denoted by $|D|$. If $B$ is a BA, then $|B|$ denotes the cardinality of the universe of $B$. If $f$ is a function, then $\operatorname{Dom}(f)$, $\operatorname{Rng}(f)$ denote the domain and range of $f$ respectively.

If $M$ is a model $\varphi\left(x_{1}, \ldots, x_{n}\right)$ is a formula in the language of $M$ and $a_{1}, \ldots, a_{n}$ belong to the universe of $M$, then $M \vDash \varphi\left[a_{1}, \ldots, a_{n}\right]$ means that $\left\langle a_{1}, \ldots, a_{n}\right\rangle$ satisfies the formula $\varphi$ in $M$.

$M \prec N$ means that $M$ is an elementary submodel of $N$.

If $M$ is a model, $P$ is a subset of the universe of $M$, then $(M, P)$ denotes the model gotten from $M$ by adding to the language of $M$ a unary predicate, to represent $P$.

A Boolean term is a term in the language of Boolean algebras.

3. The construction. We will construct an uncountable BA, in which every nowhere dense set is countable, i.e. of power $\leqslant \boldsymbol{\aleph}_{0}$. (See Definitions 3.1-3.3.) All the properties mentioned in the introduction will follow from this property.

Definition 3.1. If $a, b \in B$, then $(a, b) \stackrel{\text { def }}{=}\{c \mid c \in B$ and $a \underset{\neq}{c} \underset{\neq}{\subset} b\}$, and $[a, b] \stackrel{\text { def }}{=}\{c \mid c \in B$ and $a \subseteq c \subseteq b\}$ are called the open and respectively the closed interval with end points $a$ and $b$.

DEFINITION 3.2. If $n \geqslant 0$ and $a, b_{1}, \ldots, b_{n}, c_{1}, c_{2} \in B$, then $R\left(a, b_{1}, \ldots, b_{n}, c_{1}, c_{2}\right)$ iff $a, b_{1}, \ldots, b_{n}$ are pairwise disjoint, $c_{1} \subseteq a \cup \cup_{i=1}^{n} b_{i}, a \cup c_{1} \subseteq c_{2}$ and for every $1 \leqslant i \leqslant n,\left(c_{2}-c_{1}\right) \cap b_{i} \neq 0$.

Definition 3.3. A subset $P$ of $B$ is called nowhere dense (nwd): if for every $n>0$, $a, b_{1}, \ldots, b_{n} \in B$ such that $a, b_{1}, \ldots, b_{n}$ are pairwise disjoint and $b_{1}, \ldots, b_{n} \neq 0$, there are $c_{1}, c_{2} \in B$ such that: $R\left(a, b_{1}, \ldots, b_{n}, c_{1}, c_{2}\right)$ and $P \cap\left(c_{1}, c_{2}\right)=\varnothing$.

If $P$ is not nwd, then $P$ is called somewhere dense (swd). 
Note that the more straightforward way to define nowhere denseness would have been with $n=1$ only; but it was later noticed by Shelah that with such a definition every uncountable BA would contain an uncountable nwd set.

Definition 3.4. Let $P \subseteq B \subseteq A$. $A$ is convenient for $P, B$ (Notation: $C(A, P, B)$ ) if: for every $n \geqslant 0, a, b_{1}, \ldots, b_{n} \in A$ such that $a, b_{1}, \ldots, b_{n}$ are pairwise disjoint and for every $1 \leqslant i \leqslant n, b_{i} \neq 0$, there are $c_{1}, c_{2} \in B$ such that: $R\left(a, b_{1}, \ldots, b_{n}, c_{1}, c_{2}\right)$ and $\left(c_{1}, c_{2}\right) \cap P=\varnothing$.

Note that $C(A, P, A)$ is equivalent to $P$ is a nwd subset of $A$.

The following lemma summarizes some trivial observations.

LEMMA 3.5. (a) If $R\left(a, b_{1}, \ldots, b_{n}, c_{1}, c_{2}\right)$ and for every $1 \leqslant i<j \leqslant n: b_{i} \subseteq b_{i}^{\prime}$ and $b_{i}^{\prime} \cap b_{j}^{\prime}=b_{i}^{\prime} \cap a=0$, then $R\left(a, b_{1}^{\prime}, \ldots, b_{n}^{\prime}, c_{1}, c_{2}\right)$.

(b) If $P \subseteq B \subseteq A, D \subseteq A$ is dense in $A$ and for every $a \in A, n \geqslant 0, b_{1}, \ldots, b_{n} \in D$ $-\{0\}$ such that $a, b_{1}, \ldots, b_{n}$ are pairwise disjoint, there are $c_{1}, c_{2} \in B$ such that $R\left(a, b_{1}, \ldots, b_{n}, c_{1}, c_{2}\right)$ and $P \cap\left(c_{1}, c_{2}\right)=\varnothing$; then $C(A, P, B)$.

(c) If $\left\{A_{i} \mid i<\omega\right\}$ is an increasing chain of $B A$ 's and for every $i<\omega C\left(A_{i}, P, B\right)$, then $C\left(\cup_{i<\omega} A_{i}, P, B\right)$.

If $B$ is a BA and $R=\left\{a_{i}=b_{i} \mid i \in I\right\}$ is a list of equalities between elements of $B$, then $R$ determines a homomorphic image of $B$, namely $B / J$ where $J$ is the ideal generated by the set $\left\{a_{i} \Delta b_{i} \mid i \in I\right\}$; we will refer to this Boolean algebra as $B / R$. Let $k$ be the canonical mapping from $B$ to $B / R$; certainly if $(a=b) \in R$ then $k(a)=k(b)$.

We omit the easy proof of the following lemma.

Lemma 3.6. Let $C=\{0, x,-x, 1\}$ be a $B A$ with exactly four elements. For every $i \in I$ let $a_{i}, b_{i} \in B$, and assume that for every $i \in I, b_{i} \subseteq a_{i}$ and for every distinct $i, j \in I a_{i} \cap a_{j}=0$. Let $B_{1}$ be the free product of $B$ and $C$, and let $R=\left\{a_{i} \cap x=b_{i} \mid\right.$ $i \in I\}$. Let $k$ be the canonical homomorphism from $B_{1}$ to $B_{1} / R$; then $k \uparrow B$ is $1-1$, that is, $B$ can be regarded as a subalgebra of $B_{1} / R$.

Lemma 3.7 (Main lemma). Suppose $A$ is countable and atomless. For every $i<\omega$ let $P_{i} \subseteq B_{i} \subseteq A$, and assume $C\left(A, P_{i}, B_{i}\right)$, then there is $A_{1}$ such that $A \subsetneq A_{1}, A$ is dense in $A_{1}$, and for every $i<\omega C\left(A_{1}, P_{i}, B_{i}\right)$.

Proof. Let $x$ be an element not in $B$, and let $C=\{0, x,-x, 1\}$ be a BA with exactly four elements; we will define by induction a set of equations $R$ in the free product $A^{\prime}$ of $A$ and $C$ of the form $x \cap a=b$ where $a, b \in A$. $A_{1}$ will be $A^{\prime} / R$.

Let $T=\{a \cup(b \cap x) \cup(c-x) \mid a, b, c \in A$ and $a, b, c$ are pairwise disjoint $\}$. Note that every element of $A^{\prime}$ is represented by an element of $T$, so $T$ will represent (no doubt with repetitions) the elements of $A_{1}$. Let $\left\{s_{n} \mid n<\omega\right\}$ be a list of the following objects $A \cup T \cup\left\{\left\langle t, b_{1}, \ldots, b_{k}, i\right\rangle \mid t \in T, k \geqslant 0, b_{1}, \ldots, b_{k}\right.$ are pairwise disjoint nonzero elements of $A$, and $i<\omega\} .\left\{s_{n} \mid n<\omega\right\}$ represents the list of tasks that we will have to carry out along the definition of $R$. So if $s_{n} \in A$, it will mean that in the $n$th step we assure that $x \neq s_{n}$. Taking care of these tasks will ascertain 
that $A_{1}$ will be a proper extension of $A . s_{n} \in T$ means that in the $n$th step we have either to decide that $s_{n}=0$ or else to find $b \in A$ and to add a relation to $R$ to the effect that $b \subseteq s_{n}$. This will assure the denseness of $A$ in $A_{1}$. When $s_{n}=\left\langle t, b_{1}\right.$ $\left.\cdots b_{k}, i\right\rangle$ we will add a relation for one of the following purposes: (1) make $t \in A$, (2) make $t \cap b_{j} \neq 0$ for some $j$, (3) for some $c_{1}, c_{2} \in B_{i}$ such that $P_{i} \cap\left(c_{1}, c_{2}\right)=\varnothing$ make $R\left(t, b_{1}, \ldots, b_{k}, c_{1}, c_{2}\right)$ hold.

The induction hypothesis: after the $n$th step in the construction we have decided upon the following $n$ relations: $a_{i}^{\prime} \cap x=b_{i}^{\prime}$ where for every $0 \leqslant i<j \leqslant n, b_{i}^{\prime} \subseteq a_{i}^{\prime}$, $a_{i}^{\prime} \cap a_{j}^{\prime}=0$, and $\cup_{i=0}^{n} a_{i}^{\prime} \neq 1$. Let $a_{n}=\bigcup_{i=0}^{n} a_{i}^{\prime}$ and $b_{n}^{*}=\bigcup_{i=0}^{n} b_{i}^{\prime}$, then the relation $a_{n} \cap x=b_{n}^{*}$ is equivalent to the set of relations $\left\{a_{i}^{\prime} \cap x=b_{i}^{\prime} \mid 0 \leqslant i \leqslant n\right\}$. So it is equivalent to assume that after $n$ steps we add the relation $a_{n} \cap x=b_{n}^{*}$ where $b_{n}^{*} \subseteq a_{n} \neq 1$.

Step $n+1$. Suppose $s_{n}=a \in A$. If $a \cup a_{n} \neq 1$ let $e \in A$ such that $e \cap\left(a \cup a_{n}\right)$ $=0, e \neq 0$ and $e \cup a \cup a_{n} \neq 1$. (Remember that $A$ is atomless.) Let us add the relation $x \cap e=e$, so $a_{n+1}=a \cup e$ and $b_{n+1}^{*}=b_{n}^{*} \cup e$, so $a_{n+1} \neq 1$. Since in $A^{\prime} / R$ $e \neq 0$ this will assure that $x \neq a$. If $a \cup a_{n}=1$ then $a-a_{n} \neq 0$. Let $0 \neq e \subsetneq a-a_{n}$ and add the relation $x \cap e=0$. It is clear again that the induction hypothesis holds, and $x$ will be different from $a$.

Suppose now that $s_{n}=a \cup(b \cap x) \cup(c-x)=t \in T$. So

$$
\begin{aligned}
t & =a \cup\left(b \cap x \cap a_{n}\right) \cup\left(b \cap x-a_{n}\right) \cup\left((c-x) \cap a_{n}\right) \cup\left((c-x)-a_{n}\right) \\
& =\left[a \cup\left(b \cap b_{n}^{*}\right) \cup\left(\left(a_{n}-b_{n}^{*}\right) \cap c\right)\right] \cup\left[\left(b-a_{n}\right) \cap x\right] \cup\left[\left(c-a_{n}\right)-x\right] .
\end{aligned}
$$

So by renaming $a, b$ and $c$ we can w.l.o.g. assume that $(b \cup c) \cap a_{n}=0$. Now if $a \neq 0$ then we do not add any relation, because already $a \in A$ and $0 \neq a \subseteq t$.

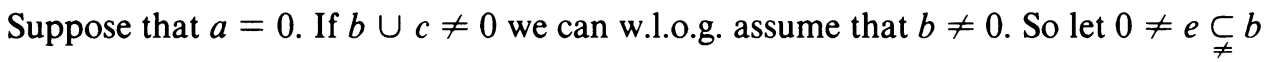
and add the relation $e \cap x=e$; we thus assure that $e \subseteq t$. It is clear that $a_{n+1}=a_{n}$ $\cup e \neq 1$, and since $e \cap a_{n}=0$, the induction hypothesis holds. If $b \cup c=0$, then $t=0$, and we do not add any relation to our list.

Suppose now that $s_{n}=\left\langle t, b_{1}, \ldots, b_{k}, i\right\rangle$ where $t \in T, b_{1}, \ldots, b_{k}$ are nonzero pairwise disjoint elements of $A$ (note that $k$ might be 0 ), and $i \in \omega$. Let $t=a \cup(b \cap x)$ $\cup(c-x)$, and as before w.l.o.g. $(b \cup c) \cap a_{n}=0$. If $\left(\cup_{i=1}^{k} b_{i}\right) \cap a \neq 0$ then $t, b_{1}, \ldots, b_{k}$ will not be a set of pairwise disjoint elements in $A_{1}$, so we do not have to add any relation. Let $d=b \cup c$. If for some $1 \leqslant j \leqslant k, b_{j} \cap d \neq 0$ let us assume w.l.o.g. that $b_{j} \cap c \neq 0$. Let $0 \neq e \supsetneqq b_{j} \cap c$ and add the relation $x \cap e=0$. It is easy to check that the induction hypothesis holds and that in $A_{1} t \cap b_{j} \neq 0$, so in $A_{1}$ $t, b_{1}, \ldots, b_{k}$ will not be pairwise disjoint. If $d=0$ then $t=a$, and since $C\left(A, P_{i}, B_{i}\right)$, we do not have to worry. Suppose now that $d \neq 0$ and for every $j d \cap b_{j}=0$. So $a, d, b_{1}, \ldots, b_{k}$ are pairwise disjoint elements of $A$ and $d, b_{1}, \ldots, b_{k} \neq 0$. Since $C\left(A, P_{i}, B_{i}\right)$, there are $c_{1}, c_{2} \in B_{i}$ such that $P_{i} \cap\left(c_{1}, c_{2}\right)=\varnothing$ and $R\left(a, d, b_{1}, \ldots, b_{k}, c_{1}, c_{2}\right)$. We are going to add a relation in order to make $R\left(t, b_{1}, \ldots, b_{k}, c_{1}, c_{2}\right)$ hold. Let us add the relations $x \cap\left(c_{1} \cap d\right)=c_{1} \cap b$ and $x \cap\left(d-c_{2}\right)=c-c_{2}$. Since $c_{1} \subseteq c_{2},\left(c_{1} \cap d\right) \cap\left(d-c_{2}\right)=0$, and since $\left(c_{2}-c_{1}\right)$ $\cap d \neq 0, a_{n+1}=a_{n} \cup\left(c_{1} \cap d\right) \cup\left(d-c_{2}\right) \neq 1$. Thus the induction hypothesis 
holds. Let us see that we have assured that $c_{1} \subseteq t \cup \cup_{i=1}^{k} b_{i}$ and $t \subseteq c_{2}$. In order to prove that $c_{1} \subseteq t \cup \cup_{i=1}^{k} b_{i}$ it is sufficient to show that $c_{1} \cap d \subseteq t$.

$$
\begin{aligned}
t & \supseteq(b \cap x) \cup(c-x) \supseteq\left[b \cap c_{1} \cap x\right] \cup\left[(c-x) \cap c_{1}\right] \\
& =\left(c_{1} \cap b\right) \cup\left(c \cap c_{1}-x\right)=\left(c_{1} \cap b\right) \cup\left[\left(c \cap c_{1}\right)-\left(c \cap c_{1} \cap x\right)\right] \\
& \supseteq\left(c_{1} \cap b\right) \cup\left[c \cap c_{1}-d \cap c_{1} \cap x\right]=\left(c_{1} \cap b\right) \cup\left(c \cap c_{1}-c_{1} \cap b\right) \\
& =\left(c_{1} \cap b\right) \cup\left(c_{1} \cap c\right)=c_{1} \cap d .
\end{aligned}
$$

In order to show that $t \subseteq c_{2}$ it is sufficient to show that $t \cap d \subseteq c_{2} \cap d$.

$$
\begin{aligned}
t \cap d & =\left(t \cap d \cap c_{2}\right) \cup\left(t \cap-c_{2}\right) \subseteq c_{2} \cup\left[t \cap\left(d-c_{2}\right)\right] \\
& =c_{2} \cup\left[b \cap x \cap\left(d-c_{2}\right)\right] \cup\left[(c-x) \cap\left(d-c_{2}\right)\right] \\
& =c_{2} \cup\left(b \cap\left(c-c_{2}\right)\right) \cup\left[(c-x) \cap\left(d-c_{2}\right)\right] \\
& =c_{2} \cup\left[c \cap\left(d-c_{2}\right)-x \cap\left(d-c_{2}\right)\right]=c_{2} \cup\left[c \cap\left(d-c_{2}\right)-\left(c-c_{2}\right)\right] \\
& =c_{2} \cup\left(\left(c-c_{2}\right)-\left(c-c_{2}\right)\right)=c_{2} .
\end{aligned}
$$

Now it is clear that $R\left(t, b_{1}, \ldots, b_{k}, c_{1}, c_{2}\right)$ will hold in $A_{1}$.

Let $R=\left\{x \cap a_{n}=b_{n}^{*} \mid n<\omega\right\}$ and let $A_{1}=A^{\prime} / R$. Since by the construction $A$ is dense in $A_{1}$, by Lemma 3.5(b) $A_{1}$ is as desired. Q.E.D.

TheOREM 3.8 (MAIN THEOREM). $\left(\diamond_{\aleph_{1}}\right)$ There is an uncountable $B A B$, such that every $n w d$ subset of $B$ is of power $\leqslant \boldsymbol{\aleph}_{0}$.

Proof. We define by induction an increasing continuous chain of BA's $\left\{B_{\alpha} \mid \alpha<\right.$ $\boldsymbol{\aleph}_{1}$ and $\alpha$ is a limit $\}$ and a sequence $\left\{P_{\alpha} \mid<\aleph_{1}\right.$ and $\alpha$ is a limit $\}$ such that: the universe of $B_{\alpha}$ is $\alpha, B_{\omega}$ is atomless and for every $\omega \leqslant \alpha B_{\omega}$ is dense in $B_{\alpha}$; for every $\alpha$ $P_{\alpha} \subseteq B_{\alpha}$ and for every $\alpha \leqslant \beta C\left(B_{\beta}, P_{\alpha}, B_{\alpha}\right)$.

Let $\left\{S_{\alpha} \mid \alpha<\boldsymbol{N}_{1}\right\}$ be the sequence assured by $\nabla_{\boldsymbol{N}_{1}}$. Let $B_{\omega}$ be an atomless BA with universe $\omega$. If $\delta$ is a limit of limit ordinals let $B_{\delta}=\cup_{\alpha<\delta} B_{\alpha}$. Suppose $B_{\alpha}$ and $P_{\beta}$, $\beta<\alpha$, have been defined. If $C\left(B_{\alpha}, S_{\alpha}, B_{\alpha}\right)$ let $P_{\alpha}=S_{\alpha}$, and otherwise let $P_{\alpha}=\varnothing$. Now by the induction hypothesis and by Lemma 3.7, there is a BA $B_{\alpha+\omega}$ with universe $\alpha+\omega$, such that for every $\beta \leqslant \alpha C\left(B_{\alpha+\omega}, P_{\beta}, B_{\beta}\right)$ and $B_{\alpha}$ is dense in $B_{\alpha+\omega}$; so the induction hypotheses hold.

Let $B=\cup_{\alpha<\kappa_{1}} B_{\alpha}$. Suppose by contradiction $P$ is a nwd uncountable subset of $B$.

Let $F=\left\{\alpha \mid \alpha<\aleph_{1}, \alpha\right.$ is a limit and $\left.\left(B_{\alpha}, \alpha \cap P\right) \prec(B, P)\right\}$; then $F$ is closed and unbounded. Let $S=\left\{\alpha \mid P \cap \alpha=S_{\alpha}\right\}$, then $S$ is stationary; so $S \cap F \neq \varnothing$. Let $\alpha_{0} \in S \cap F$. Since $P$ is nwd in $B$, and $\left(B_{\alpha_{0}}, P \cap \alpha_{0}\right) \prec(B, P), P \cap \alpha_{0}$ is nwd in $B_{\alpha_{0}}$, thus $P \cap \alpha_{0}=P_{\alpha_{0}}$. Let $a \in P-P_{\alpha_{0}}$, so there are $c_{1}, c_{2} \in B_{\alpha_{0}}$, such that $R\left(a, c_{1}, c_{2}\right)$, i.e. $c_{1} \subseteq a \subseteq c_{2}$, and $P_{\alpha_{0}} \cap\left(c_{1}, c_{2}\right)=\varnothing$. But then: $\left(B_{\alpha_{0}}, P_{\alpha_{0}}\right) \vDash \forall x(P(x) \rightarrow x \notin$ $\left.\left(c_{1}, c_{2}\right)\right)$, whereas $(B, P) \vDash P(a) \wedge a \in\left(c_{1}, c_{2}\right)$. This contradicts the fact that $\left(B_{\alpha_{0}}, P_{\alpha_{0}}\right) \prec(B, P)$. Q.E.D.

REMARKS. (a) In the construction we can also take care that for every $b \in B-\{0\}$ $|(0, b)|=\boldsymbol{\aleph}_{1}$. (b) Let us denote by $\prod_{i \in I} B_{i}$ the full direct product of $\left\{B_{i} \mid i \in I\right\}$ and by $\Sigma_{i \in I} B_{i}$ the weakest direct product of $\left\{b_{i} \mid i \in I\right\}$. 
Let $\left\{B_{i} \mid i \in I\right\}=\widetilde{\mathscr{y}}$ be a family of BA's. $B$ is called a good product for $\mathscr{F}$, if there is a countable or finite $J \subseteq I$ such that: $\sum_{i \in J} B_{i} \subseteq B \subseteq \Pi_{i \in J} B_{i}$, and $B / K$ is countable or finite, where $K$ is the ideal of $B$ generated by $\cup_{i \in J} B_{i}$. The construction can be modified to yield a family $\left\{B_{i} \mid i<2^{\aleph_{1}}\right\} \stackrel{\text { def }}{=} \mathscr{F}$, such that for every $i<2^{\aleph_{1}} B_{i}$ is uncountable, and whenever $B$ is a good product for $\mathscr{F}, B$ does not contain uncountable nwd sets.

Note that this implies that for every $i<j<2^{\boldsymbol{N}_{1}}$ and every BA $C$ which is a homomorphic image or embeddable in $B_{i}$, and which is a homomorphic image or embeddable in $B_{j}, C$ is countable or finite.

Definition 3.9. A BA $B$ is called partly concentrated if: (1) $B$ is atomless, and $|B|=\boldsymbol{\aleph}_{1}$; (2) $I(B)$ is a prime ideal of $B$; (3) if $P \subseteq B,|P|=\boldsymbol{\aleph}_{1}$, then there are $a \in I(B), b \in B-I(B)$ such that $a \subseteq b$, and for every $a_{1} \in I(B), b_{1} \in B-I(B)$ such that $a \subseteq a_{1} \subseteq b_{1} \subseteq b: P \cap\left(a_{1}, b_{1}\right) \neq \varnothing$.

In a method similar to 3.8 one can prove the following theorem.

THEOREM 3.10. $\left(\diamond_{\aleph_{1}}\right)$ There is a partly concentrated $B A$.

Theorem 3.11 is due to Shelah; it shows that gap-two theorems are not true for strongly concentrated BA's.

THEOREM 3.11. Let $B$ have the property that every uncountable set contains distinct elements $a, b, c$ such that $a \cap b=c$; then $|B| \leqslant \boldsymbol{\aleph}_{1}$.

Proof. Let us assume by contradiction that $B$ has the above property but $|B|>\boldsymbol{\aleph}_{1}$. W.l.o.g. $|B|=\boldsymbol{\aleph}_{2}$. It is obvious that every uncountable subset of $B$ contains distinct elements $a, b, c$ such that $a \cup b=c$.

We first show that every ideal in $B$ is countably generated. Suppose by contradiction that $I$ is not countably generated. Then there is a sequence $\left\{a_{i} \mid i<\boldsymbol{\aleph}_{1}\right\} \subseteq I$ such that for every $j_{1}<\cdots<j_{n}<i<\aleph_{1}: a_{i} \nsubseteq \cup_{k=1}^{n} a_{j_{k}}$. Let $a_{i}, a_{j}, a_{k}$ be distinct elements in the above sequence such that $a_{i} \cup a_{j}=a_{k}, i, j<k$, is certainly impossible. If however $k<j$, then $a_{j} \subseteq a_{k}$ contradicts again the property of the sequence. Hence every ideal of $B$ is countably generated.

Let $\left\{B_{i} \mid i<\aleph_{2}\right\}$ be a strictly increasing continuous sequence of subalgebras of $B$, such that for every $i\left|B_{i}\right| \leqslant \boldsymbol{\aleph}_{1}$, and let $a_{i} \in B_{i+1}-B_{i}$. Let $C=\left\{i<\boldsymbol{\aleph}_{2} \mid \operatorname{cf}(i)=\boldsymbol{\aleph}_{1}\right\}$. For every $i \in C$, let $I_{i}=\left\{b \in B_{i} \mid b \subseteq a_{i}\right\}$ and $F_{i}=\left\{b \in B_{i} \mid a_{i} \subseteq b\right\}$. Clearly $I_{i}$ and $F_{i}$ are respectively an ideal and a filter in $B_{i}$, and so they are countably generated. Hence there is $\alpha_{i}<i$ such that both $I_{i}$ and $F_{i}$ are generated by subsets of $B_{\alpha_{i}}$.

By Fodor's theorem there is an uncountable set $D \subseteq C$ and $\alpha<\boldsymbol{N}_{2}$ such that for every $i \in D \alpha_{i}=\alpha$.

Let $i, j, k$ be distinct elements of $D$ such that $a_{i} \cup a_{j}=a_{k}$. Since $a_{i}, a_{j} \subseteq a_{k}$ there are $b, c \in B_{\alpha}$ such that $a_{i} \subseteq b \subseteq a_{k}$ and $a_{j} \subseteq c \subseteq a_{k}$; this can be proved by distinguishing between the cases when $i, j<k$ or $i<k<j$ or $k<i<j$. But then $b \cup c=a_{k}$ which means that $a_{k} \in B_{\alpha}$, a contradiction. Q.E.D. 


\section{Properties of strongly concentrated BA's.}

Definition 4.1. $B$ is called strongly concentrated (SC), if $|B|=\boldsymbol{\aleph}_{1}, B$ is atomless, and $B$ does not contain uncountable nwd subsets.

Let $I(B)=\left\{b \mid b \in B\right.$ and $\left.|\{a \mid B \ni a \subseteq b\}| \leqslant \boldsymbol{\aleph}_{0}\right\}$.

LEMMA 4.2. If $B$ is $S C$, then $|I(B)| \leqslant \boldsymbol{\aleph}_{0}$.

Proof. Suppose by contradiction $|I(B)|=\boldsymbol{\aleph}_{1}$; then for every $P \subseteq I(B)$ : if $|P| \leqslant \aleph_{0}$, then there is $a \in I(B)$, such that for no $b \in P, a \subseteq b$. So $I(B)$ contains a sequence $C=\left\{c_{i} \mid i<\boldsymbol{\aleph}_{1}\right\}$ such that for every $i<j<\boldsymbol{\aleph}_{1} c_{j} \nsubseteq c_{i}$. Since $|C|=\boldsymbol{\aleph}_{1}$, it is swd. Let $n>0, a, b_{1}, \ldots, b_{n} \in B$ be such that: $a, b_{1}, \ldots, b_{n}$ are pairwise disjoint, $b_{1}, \ldots, b_{n} \neq 0$, and for every $d_{1}, d_{2} \in B$ : if $R\left(a, b_{1}, \ldots, b_{n}, d_{1}, d_{2}\right)$, then $\left(d_{1}, d_{2}\right) \cap C$ $\neq \varnothing$. For every $1 \leqslant i \leqslant n$ let $\left\{b_{i}^{j} \mid j<\omega\right\}$ be a sequence such that for every $j<k<\omega \quad b_{i} \supsetneqq b_{i}^{j} \supsetneqq b_{i}^{k}$. Let $d_{j}=a \cup \cup_{i=1}^{n} b_{i}^{j} ;$ then for every $j<\omega$ $R\left(a, b_{1}, \ldots, b_{n}, d_{j+1}, d_{j}\right)$. So let $c_{\alpha_{j}} \in\left(d_{j+1}, d_{j}\right) \cap C$. If $j<k<\omega$, then $c_{\alpha_{j}} \subseteq c_{\alpha_{k}}$. On the other hand $\left\{\alpha_{j} \mid j<\omega\right\}$ is not a decreasing sequence, so for some $k>j$ $\alpha_{k}>\alpha_{j}$, but by the choice of $C, c_{\alpha_{k}} \nsubseteq c_{\alpha_{j}}$, a contradiction. Q.E.D.

THEOREM 4.3. Suppose B is strongly concentrated, then:

(a) (Shelah) $B$ has just $2^{\aleph_{0}}$ lower or upper subsemilattices.

(b) Every ideal of $B$ is countably generated (as an ideal). Every subalgebra of $B$ is generated by an ideal and a countable set.

(c) $B$ is retractive; that is, if $I$ is an ideal in $B$, then there is a subalgebra $A$ of $B$, such that for every $b \in B|A \cap b / I|=1$.

(d) (Shelah, Rubin) There are just $2^{\aleph_{0}}$ order preserving functions from $B$ to $B$. ( $f$ : $B \rightarrow B$ is order preserving, if whenever $a, b \in B$ and $a \subseteq b ; f(a) \subseteq f(b)$.)

(e) If $I(B)=\{0\}$, then $B$ does not contain 1-1 or onto endomorphism other than the identity.

REMARKS. (1) (a) improved the result of the author that $B$ has just $2^{\aleph_{0}}$ subalgebras. It answers a question of W. Rautenberg.

(2) (d) was first proved by Shelah for BA's that have a property stronger than strong concentration. The author using a similar method proved it for SC BA's. However Bonnet [B1] constructed BA's having the properties (d) and (e) assuming $\mathrm{CH}$ only.

Proof. (a) We will prove that every lower subsemilattice of $B$ can be represented as the union of countably many closed intervals. (Note that for every $a \in B$ $\{a\}=[a, a]$ is a closed interval.) Suppose by contradiction that $L$ is a counterexample. Let $\left\{\left[a_{i}, b_{i}\right] \mid i<\boldsymbol{\aleph}_{1}\right\}$ be an enumeration of all closed intervals which are contained in $L$. Since $L$ is not a union of $\leqslant \boldsymbol{\aleph}_{0}$ intervals, we can choose a sequence $C \stackrel{\text { def }}{=}\left\{c_{i} \mid i<\boldsymbol{\aleph}_{1}\right\}$ with the following properties: for every $i<\boldsymbol{\aleph}_{1}: c_{i} \in L-$ $\cup_{j<i}\left[a_{j}, b_{j}\right]$; for every $i<j<\boldsymbol{\aleph}_{1}: c_{i} \triangle c_{j} \notin I(B),|C|=\boldsymbol{\aleph}_{1}$, so there are $n>0$, $a, b_{1}, \ldots, b_{n} \in B$ such that: $a, b_{1}, \ldots, b_{n}$ are pairwise disjoint, $b_{1}, \ldots, b_{n} \neq 0$; and for every $d_{1}, d_{2} \in B$, if $R\left(a, b_{1}, \ldots, b_{n}, d_{1}, d_{2}\right)$, then $C \cap\left(d_{1}, d_{2}\right)=\varnothing$. Let $b \in B$ 
be such that $a \subseteq b, b \subseteq a \cup \cup_{i=1}^{n} b_{i}$, and for every $1 \leqslant i \leqslant n b_{i}-b, b \cap b_{i} \neq 0$. We will show that $[a, b] \subseteq L$. Let $d \in[a, b]$. For every $1 \leqslant i \leqslant n, j=1,2$, let $d_{i}^{j} \subseteq b_{i}-d, d_{i}^{j} \neq 0$, and $d_{i}^{1} \cap d_{i}^{2}=0$. Let $d^{j}=d \cup \cup_{i=1}^{n} d_{i}^{j}, j=1,2$. Then $R\left(a, b_{1}, \ldots, b_{n}, d, d^{j}\right), j=1,2$. So let $c^{j} \in\left(d, d^{j}\right) \cap C, j=1,2 . d=c^{1} \cap c^{2}$, and $c^{1}, c^{2} \in L$, so $d \in L$.

Since $|[a, b] \cap C|>1, b-a \notin I(B)$; so $|[a, b] \cap C|=\boldsymbol{\aleph}_{1}$. But $[a, b] \subseteq L$, so for some $i<\aleph_{1}[a, b]=\left[a_{i}, b_{i}\right]$, but then $C \cap[a, b] \subseteq\left\{c_{j} \mid j<i\right\}$, so $|C \cap[a, b]|$ $\leqslant \boldsymbol{\aleph}_{0}$, a contradiction. So every lower subsemilattice of $B$ is the union of countably many closed intervals; so $B$ has $\aleph_{1}^{\aleph_{0}}=2^{\aleph_{0}}$ lower subsemilattices.

A similar argument holds for upper subsemilattices. Q.E.D.

(b) Let $I$ be an ideal in $B$; then $I$ is a sublattice of $B$, so there are $a_{i}, b_{i} \in B$, $a_{i} \subseteq b_{i}, i<\omega$, such that $I=\cup_{i<\omega}\left[a_{i}, b_{i}\right]$, so $I$ is generated as an ideal by the set $\left\{b_{i} \mid i<\omega\right\}$. Let $A$ be a subalgebra of $B$. Let $A=\cup_{i<\omega}\left[a_{i}, b_{i}\right]$ and $a_{i} \subseteq b_{i}$. Let $I$ be the ideal generated by $\left\{b_{i}-a_{i} \mid i<\omega\right\}$; then $A$ is generated by $I \cup\left\{b_{i} \mid i<\omega\right\}$.

(c) We first prove that if $I \subseteq B$ is a dense ideal, then $|B / I| \leqslant \aleph_{0}$. If not, let $P \subseteq B$ be such that for every $b \in B|b / I \cap P|=1$. Since $|P|=\boldsymbol{\aleph}_{1}, P$ is swd. Let $a, b_{1}, \ldots, b_{n}$ exemplify this fact. For every $1 \leqslant i \leqslant n$ let $a \neq b_{1}^{\prime} \subseteq b_{i}$ and $b_{i}^{\prime} \in I$. So $\left|P \cap\left[a, a \cup \cup_{i=1}^{n} b_{i}^{\prime}\right]\right| \geqslant 2$, contradicting the choice of $P$. So $|B / I| \leqslant \boldsymbol{\aleph}_{0}$.

Secondly the reader can easily check: $(*)$ if $A$ is a $\mathrm{BA}, I \subseteq A$ is an ideal, and $|A / I| \leqslant \boldsymbol{\aleph}_{0}$, then there is a subalgebra $A_{1}$ of $A$ such that for every $a \in A$ $\left|A_{1} \cap a / I\right|=1$.

Let $I$ be an ideal in $B$. Let $J=\{a \mid a \in B$ and for every $b \in I a \cap b=0\}$; then $J$ is an ideal and $I \cup J$ generates a dense ideal $I^{\prime}$. So $\left|B / I^{\prime}\right| \leqslant \boldsymbol{\aleph}_{0}$. Let $A_{1}$ be the subalgebra assured by (*). Let $A_{2}$ be the algebra generated by $J \cup A_{1}$. We prove that $A_{2}$ is as required. If not, there is $a$ such that $0 \neq a \in A_{2} \cap I$. W.l.o.g. $a=b \cap c$ where $b \in J$ or $-b \in J$ and $c \in A_{1}$. But $b \in J$ is impossible, so $-b \in J$; so $c \cap-b \in I^{\prime}$ and $c \cap b \in I^{\prime}$, so $c \in I^{\prime}$. But by choice of $A_{1}$, this is impossible. So (c) is proved.

(d) Let us first mention that an SC BA does not contain uncountable antichains. (See Theorem 4.6(c).)

Let $f: B \rightarrow B$ be an order preserving function. We will prove: $(*)$ there is a sequence $\left\{\left\langle a_{i}, b_{i}, c_{i}, d_{i}\right\rangle \mid i<\omega\right\}$ such that: $B=\cup_{i<\omega}\left[a_{i}, b_{i}\right]$, and for every $x \in$ $\left[a_{i}, b_{i}\right], f(x)=c_{i} \cup\left(d_{i} \cap x\right)$.

Suppose $f$ is a counterexample to $(*)$. In a way similar to what was done in part (a), one can choose an uncountable subset $C$ of $B$ with the following properties: (1) If $a, b, c, d \in B$ are such that for every $x \in[a, b], f(x)=c \cup(d \cap x)$; then $\mid C \cap$ $[a, b] \mid \leqslant \boldsymbol{\aleph}_{0} ;(2)$ if $c_{1}, c_{2} \in C$ and $c_{1} \neq c_{2}$, then $c_{1} \Delta c_{2} \notin I(B)$.

Let $a, b_{1}, \ldots, b_{n}$ exemplify the fact that $C$ is swd. We will first find $a^{\prime}, b_{1}^{\prime}, \ldots, b_{n}^{\prime}$ and $\sigma_{1}, \sigma_{2}, \sigma_{3} \subseteq\{1, \ldots, n\}$ such that: $a \subseteq a^{\prime} \subseteq a \cup \cup_{i=1}^{n} b_{i}, 0 \neq b_{i}^{\prime} \subseteq b_{i}, b_{1}^{\prime} \cap a^{\prime}=0$, $\sigma_{1} \cup \sigma_{2} \cup \sigma_{3}=\{1, \ldots, n\}$, and for every $x \in\left[a^{\prime}, a^{\prime} \cup \cup_{i=1}^{n} b_{i}\right]$ and $1 \leqslant i \leqslant n$ : if $i \in \sigma_{1}$ then $f(x) \supseteq b_{i}^{\prime}$, if $i \in \sigma_{2} f(x) \cap b_{i}^{\prime}=0$, and if $i \in \sigma_{3}$ then $f(x) \cap b_{i}^{\prime}=x \cap b_{i}^{\prime}$.

Let $\sigma_{1} \subseteq\{1, \ldots, n\}$ be a maximal set with the property that there is $c \in B$ such that: $a \subseteq c \subseteq a \cup \cup_{i=1}^{n} b_{i}$, for every $1 \leqslant i \leqslant n b_{i} \cap c \neq 0 \neq b_{i}-c$, and for every $i \in \sigma_{1}(f(c)-c) \cap b_{i} \neq 0$. For every $i \in \sigma_{1}$ let $b_{i}^{1}=(f(c)-c) \cap b_{i}$; for every $i \in\{1, \ldots, n\}-\sigma_{1}$ let $b_{i}^{1}=b_{i}-c$. Let $\sigma_{2} \subseteq\{1, \ldots, n\}$ be a maximal set with the 
property: there is $c_{1} \in B$ such that $c_{1} \in\left[c, c \cup \cup_{i=1}^{n} b_{i}^{1}\right]$, for every $i \in\{1, \ldots, n\}$ $c_{1} \cap b_{i}^{1} \neq 0$, and for every $i \in \sigma_{2}, f\left(c_{1}\right) \cap b_{i}^{1} \subsetneq c_{1} \cap b_{i}^{1}$. Let $a^{\prime}=c \cup\left(f\left(c_{1}\right) \cap\right.$ $\left.\cup_{i \in \sigma_{2}} b_{i}^{1}\right)$, and let $b_{i}^{\prime}=\left(c_{1}-a^{\prime}\right) \cap b_{i}^{1}, i=1, \ldots, n$.

It is easily seen that $a^{\prime}, b^{\prime}, \ldots, b_{n}^{\prime}, \sigma_{1}, \sigma_{2}$ and $\sigma_{3} \stackrel{\text { def }}{=}\{1, \ldots, n\}-\sigma_{1}-\sigma_{2}$ are as desired.

It is also clear that $a^{\prime}, b_{1}^{\prime}, \ldots, b_{n}^{\prime}$ exemplify the fact that $C$ is swd. So let us rename $a^{\prime}, b_{1}^{\prime}, \ldots, b_{n}^{\prime}$ by $a, b_{1}, \ldots, b_{n}$ respectively. Let $C_{1}=C \cap\left[a, a \cap \cup_{i=1}^{n} b_{i}\right]$. Suppose first (**): $\left|\left\{f(c)-\bigcup_{i=1}^{n} b_{i} \mid c \in C_{1}\right\}\right|=\boldsymbol{\aleph}_{1}$. Then there is a $C_{2} \subseteq C_{1}$ such that $\left|C_{2}\right|=\boldsymbol{\aleph}_{1}$, and for every $c_{1}, c_{2} \in C_{2}$ : if $c_{1} \neq c_{2}$, then $f\left(c_{1}\right)-\bigcup_{i=1}^{n} b_{i} \neq f\left(c_{2}\right)-$ $\cup_{i=1}^{n} b_{i}$. Let $P=\left\{\left(c \cap \cup_{i=1}^{n} b_{i}\right) \cup\left(-f(c)-\cup_{i=1}^{n} b_{i}\right) \mid, c \in C_{2}\right\}$; then $P$ is an uncountable antichain. But this is impossible, so $(* *)$ does not hold. So there is $C_{3} \subseteq C_{1}$ such that $\left|C_{3}\right|=\boldsymbol{\aleph}_{1}$ and for every $c_{1}, c_{2} \in C_{3}: f\left(c_{1}\right)-\cup_{i=1}^{n} b_{i}=f\left(c_{2}\right)-$ $\bigcup_{i=1}^{n} b_{i}$. Let $e_{1}=\cup_{i \in \sigma_{1}} b_{i}, e_{3}=\cup_{i \in \sigma_{3}} b_{i}, c_{0} \in C_{3}$ and $d=f\left(c_{0}\right)-\bigcup_{i=1}^{n} b_{i}$; then, for every $x \in C_{3}, f(x)=\left(e_{1} \cup d\right) \cup\left(e_{3} \cap x\right)$. Let $a^{\prime}, b_{1}^{\prime}, \ldots, b_{k}^{\prime}$ exemplify the fact that $C_{3}$ is swd. It is easy to see that if $x \in\left(a^{\prime}, a^{\prime} \cup \cup_{i=1}^{k} b_{i}^{\prime}\right)$ and for every $1 \leqslant i \leqslant k$, $x \cap b_{i}^{\prime} \neq 0 \neq b_{i}^{\prime}-x$, then $f(x)=\left(e_{1} \cup d\right) \cup\left(e_{3} \cap x\right)$. Let $0 \neq b_{i}^{1} \subsetneq b_{i}^{2} \subsetneq b_{i}^{\prime}, i=$ $1, \ldots, k$, and let $a^{\prime \prime}=a^{\prime} \cup \cup_{i=1}^{k} b_{i}^{1}$ and $b^{\prime \prime}=\cup_{i=1}^{k}\left(b_{i}^{2}-b_{i}^{1}\right)$; then, for every $x \in$ $\left[a^{\prime \prime}, a^{\prime \prime} \cup b^{\prime \prime}\right], f(x)=\left(e_{1} \cup d\right) \cup\left(e_{3} \cap x\right)$ and $\left|C_{3} \cap\left[a^{\prime \prime}, b^{\prime \prime}\right]\right|=\aleph_{1}$. This contradicts the choice of $C$. So we proved (*). It follows trivially that $\mid\{f \mid f: B \rightarrow B$ is order preserving $\} \mid \leqslant 2^{\aleph_{0}}$.

Question. For which formulas $\varphi\left(x_{1}, \ldots, x_{n}\right)$ are there just $2^{\aleph_{0}} \varphi$ preserving functions?

(e) REMARKS. (1) Baumgartner noted that if $B$ does not contain uncountable antichains and $I(B)=\{0\}$ then $B$ is rigid. We note in a similar way, that such a $B$ does not have 1-1 order preserving functions other than the identity.

(2) Every BA with more than two elements has an onto order preserving function different from the identity. For, certainly it is true for finite BA's; so let $|B| \geqslant \boldsymbol{\aleph}_{0}$, let $\left\{a_{i} \mid i<\omega\right\}$ be a set of pairwise disjoint nonzero elements of $B$. Define $f(x)$ $=x$, if $\left\{i \mid x \cap a_{i} \neq 0\right\}$ is infinite; otherwise define $f(x)=x-a_{i}$ where $i$ $=\max \left(\left\{j \mid a_{j} \cap x \neq 0\right\}\right) . f$ is as desired. Note also that $f$ preserves disjointness.

(3) Bonnet [B1] proved that if $B$ is an interval algebra without 1-1 endomorphisms except the identity, then $B$ does not have onto endomorphisms except the identity. It was noticed by Monk and Loats that this fact is true for every retractive BA. In fact it is true for every retractive algebra.

Proof of (e). Suppose $f: B \rightarrow B$ is 1-1 and order preserving. Assume by contradiction $f(a) \neq a$.

Case 1: $a-f(a) \neq 0$. Let $P=\{x \cup[f(a)-f(x)] \mid x \subseteq a-f(a)\}$, then $P$ is an uncountable antichain. By Theorem 4.6(c) this is a contradiction.

Case 2: $f(a) \supsetneqq a$. In this case $\{x \cup(-f(x) \cap-f(a)) \mid x \in(a, f(a))\}$ is an uncountable antichain, and again we reach a contradiction.

Suppose $B$ is retractive, and does not have 1-1 endomorphism except the identity. Let $f$ be an onto endomorphism and $I=\operatorname{ker}(f)$, let $A \subseteq B$ be a subalgebra of $B$ such that for every $b \in B,|A \cap b / I|=1$ then $f \uparrow A$ is an isomorphism between $A$ 
and $B$. So, $(f \uparrow A)^{-1}$ is a $1-1$ endomorphism from $B$ to $B$. So $(f \uparrow A)^{-1}=\mathrm{Id}$, hence $f=$ Id. Q.E.D.

If $B$ is a BA and $\varphi\left(x_{1}, \ldots, x_{n}\right)$ is a formula in the language of BA's, let $B \uparrow \varphi$ be the structure $M \stackrel{\text { def }}{=}\left\langle A, R^{M}\right\rangle$ where $A$ is the universe of $B$ and $R^{M}=\left\{\left\langle b_{1}, \ldots, b_{n}\right\rangle \mid B \vDash\right.$ $\left.\varphi\left[a_{1}, \ldots, a_{n}\right]\right\}$.

Questions. Suppose $B$ is SC. (1) For which formulas $\varphi B \uparrow \varphi$ is retractive.

(2) For which $\varphi$ 's $B i \varphi$ does not have 1-1 endomorphisms other than the identity, and for which $\varphi$ 's $B$ | $\varphi$ does not have onto endomorphisms other than the identity.

Definition 4.4. A configuration is a quantifier free, consistent complete (in the set of quantifier free formulas) type in the language of BA's, and with variables $\left\{x_{i} \mid i \in I\right\}$ where $|I| \leqslant \boldsymbol{\aleph}_{0}$.

Let $B$ be a $\mathrm{BA}, L$ a configuration and $P \subseteq B ; L$ appears in $P$, if for some $\left\{a_{i} \mid I<\alpha\right\} \subseteq P\left\{a_{i} \mid i<\alpha\right\}$ realizes $L$. If $L$ does not appear in $P$, then we say that $P$ is $L$-free.

$L$ is called a good configuration if there is a linear ordering $<$ of $I$, such that for every $i_{1}<i_{2}<\cdots<i_{k} \in I$ and a term $\tau\left(x_{1}, \ldots, x_{k-1}\right):\left(\cup_{j=1}^{k} x_{i}=1\right) \notin L$, $\left(\cap_{j=1}^{k} x_{i_{j}}=0\right) \notin L$ and $\left(\tau\left(x_{i_{1}}, \ldots, x_{i_{k-1}}\right)=x_{i_{k}}\right) \notin L$.

The reader can easily ascertain the following observation.

Observation 4.5. For every uncountable BA $B$ there is an uncountable subset $P$ of $B$, such that every configuration that appears in $P$ is good.

EXAMPLES. The following configurations are good: (1) $\left\{x_{i} \mid i<\omega\right\}$ is an independent set; (2) $\left\{x_{r} \subsetneq x_{q} \mid r, q\right.$ are rationals and $\left.r<q\right\}$; (3) $x_{1}=x_{2} \cap x_{3}, x_{2} \neq x_{3} \neq x_{1}$ $\neq 0$ and $x_{2} \cup x_{3} \neq 1$.

Theorem 4.6. (a) Let $A$ be an atomless $B A, L$ be a good configuration with order type $\leqslant \omega$ and $P \subseteq A$ be an $L$-free subset of $A$; then $P$ is nwd. (b) If $B$ is $S C, L$ is a good configuration with order type $\leqslant \omega$ and $P \subseteq B$ is uncountable, then $L$ appears in $P$. (c) If $B$ is $S C$, then $B$ does not contain uncountable chains or antichains.

Proof. (b) is a trivial corollary of $A$, and (c) is a trivial corollary of (b).

Proof of (a). We prove that if $P \subseteq A$ is swd, and $L$ is a good configuration then $L$ appears in $P$. W.l.o.g., $L$ is in the variables $\left\{x_{i} \mid i<\omega\right\}$. Let $a, b_{1}, \ldots, b_{m}$ exemplify the fact that $P$ is swd. We now define by induction a sequence $\left\{p_{i} \mid i<\omega\right\} \subseteq P$ with the following induction hypotheses: Suppose $p_{0}, \ldots, p_{n-1}$ have been defined $n>0$, and let $\left\{r_{1}, \ldots, r_{l}\right\}=\operatorname{At}\left(\operatorname{cl}\left(\left\{p_{0}, \ldots, p_{n-1}\right\}\right)\right)$; then:

(1) for every $i, 0 \leqslant i<n: a \subseteq p_{i} \subseteq a \cup \cup_{i=1}^{m} b_{i}$;

(2) for every $i, 0 \leqslant i<n$, and for every $j, 1 \leqslant j \leqslant m: r_{i} \cap b_{j} \neq 0$;

(3) $\left\langle p_{0}, \ldots, p_{n-1}\right\rangle$ realizes $L i\left\{x_{i} \mid i<n\right\}$.

We first construct $p_{0}$. Let $p_{0} \in P \cap\left[a, a \cup \cup_{i=1}^{m} b_{i}\right]$ be such that for every $1 \leqslant i \leqslant m, b_{i} \cap p_{0} \neq 0 \neq b_{i}-p_{0}$. Clearly, the induction hypotheses for $n=1$ are satisfied.

Suppose $p_{0}, \ldots, p_{n-1}$ have been defined, and $n>0$. Let $\left\{r_{1}, \ldots, r_{l}\right\}$ be as above. W.1.o.g. $r_{1}=\bigcap_{i<n} p_{i}$, and $r_{l}=\bigcap_{i<n}-p_{i}$. For every $1 \leqslant i \leqslant l$, let $r_{i}=$ $\tau_{i}\left(p_{0}, \ldots, p_{n-1}\right)$, and let us denote the term $\tau_{i}\left(x_{0}, \ldots, x_{n-1}\right)$ by $y_{i}$. Let $\sigma_{1}=\left\{i \mid\left(y_{i} \subseteq\right.\right.$ $\left.\left.x_{n}\right) \in L\right\}, \sigma_{2}=\left\{i \mid\left(y_{i} \cap x_{n}=0\right) \in L\right\}$ and $\sigma_{3}=\left\{i \mid\left(y_{i} \cap x_{n} \neq 0 \neq y_{i}-x_{n}\right) \in L\right\}$. 
Since for no term $\tau,\left(\tau\left(x_{0}, \ldots, x_{n-1}\right)=x_{n}\right) \in L, \sigma_{3} \neq \varnothing$. Since $\left(\bigcap_{i \leqslant n} x_{i} \neq 0\right) \in L$, $1 \notin \sigma_{2} ;$ and since $\left(\cup_{i \leqslant n} x_{i} \neq 1\right) \in L, l \notin \sigma_{1}$.

For every $i \in \sigma_{3}$ and $1 \leqslant j \leqslant m$, let $0 \neq d_{i j}^{1} \subsetneq d_{i j}^{2} \subsetneq r_{i} \cap b_{j}$. Let

$$
c_{1}^{n}=a \cup\left(\bigcup_{i \in \sigma_{1}} r_{i}\right) \cup \bigcup\left\{d_{i j}^{1} \mid i \in \sigma_{3} \text { and } 1 \leqslant j \leqslant m\right\} \text {, }
$$

and

$$
c_{2}^{n}=a \cup\left(\bigcup_{i \in \sigma_{1}} r_{i}\right) \cup \bigcup\left\{d_{i j}^{2} \mid i \in \sigma_{3} \text { and } 1 \leqslant j \leqslant m\right\} .
$$

We first show that $R\left(a, b_{1}, \ldots, b_{m}, c_{1}^{n}, c_{2}^{n}\right)$ holds. In order to show that $c_{1}^{n} \subseteq a \cup$ $\cup_{i=1}^{m} b_{i}$, it suffices to show that for every $i \in \sigma_{1}, r_{i} \subseteq a \cup \cup_{i=1}^{m} b_{i}$. But since $l \notin \sigma_{1}$, there is at least one $j, 0 \leqslant j<n$, such that $\left(y_{i} \subseteq x_{j}\right) \in L$. So $r_{i} \subseteq p_{j} \subseteq a \cup \cup_{t=1}^{m} b_{t}$. Certainly $c_{2}^{n} \subseteq c_{1}^{n} \cup a$. $\sigma_{3} \neq \varnothing$, so let $\beta \in \sigma_{3}$. Let $1 \leqslant j \leqslant m$; then $\left(c_{2}^{n}-c_{1}^{n}\right) \cap b_{j} \supseteq$ $d_{\beta j}^{2} \neq 0$. So we proved that $R\left(a, b_{1}, \ldots, b_{m}, c_{1}^{n}, c_{2}^{n}\right)$ holds.

Let $p_{n} \in P \cap\left[c_{1}^{n}, c_{2}^{n}\right]$. Since $a \subseteq c_{1}^{n} \subseteq p_{n} \subseteq c_{2}^{n} \subseteq a \cup \cup_{i=1}^{m} b_{i}$, condition (1) of the induction hypotheses holds. If $r \in \operatorname{At}\left(\operatorname{cl}\left(\left\{p_{0}, \ldots, p_{n}\right\}\right)\right)$, then either: $r \in\left\{r_{1}, \ldots, r_{l}\right\}$ and then for every $1 \leqslant j \leqslant m r \cap b_{j} \neq 0$; or else there is $\beta \in \sigma_{3}$, such that $r \supseteq$ $\cup_{j=1}^{m} d_{\beta j}^{1}$, or $r \supseteq \cup_{j=1}^{m}\left(r_{\beta} \cap b_{j}-d_{\beta j}^{2}\right)$, so in both cases for every $1 \leqslant j \leqslant m, r \cap b_{j}$ $\neq 0$. Hence, condition (2) of the induction hypotheses holds. It is clear that for every $1 \leqslant i \leqslant l: p_{n} \supseteq r_{i}$ iff $\left(x_{n} \supseteq y_{i}\right) \in L$, and $r_{i} \cap p_{n} \neq 0 \neq r_{i}-p_{n}$ iff $\left(y_{i} \cap x_{n} \neq 0 \neq y_{i}\right.$ $\left.-x_{n}\right) \in L$; so $\left\langle p_{0}, \ldots, p_{n}\right\rangle$ realizes $L \uparrow\left\{x_{0}, \ldots, x_{n}\right\}$. Thus (a) has been proved.

REMARK. Remember that by [BaK], and SC BA is embeddable in $\langle P(\omega), \cup, \cap$, $-, \varnothing, \omega\rangle$.

Question. Let $B$ be SC and $P \subseteq B$ be uncountable. Does every good configuration appear in $P$ ?

THEOREM 4.7. Let $B$ be a partly concentrated $B A$. Then: (1) $B$ is an $A J, \aleph_{1}-I P B A$ with just $\aleph_{1}$ lower or upper subsemilattices.

(2) $I(B)$ is an $A J$ lattice and it is $\aleph_{1}-I P$ lower semilattice.

Proof. Similar to the arguments previously presented in this section.

5. Some facts about interval algebras. If $\langle J,<\rangle$ is a linear ordering let $J^{+}=J \cup$ $\{-\infty, \infty\}$ (we assume that $-\infty, \infty \notin J$ ); we define the ordering on $J^{+}$in the obvious way. If $a, b \in J^{+}$let $(a, b]=\{x \mid x \in J$ and $a<x \leqslant b\}$. Note that $-\infty, \infty$ $\notin(a, b]$. Every element $a$ of the interval algebra $B(J)$ (see Definition 1.2) can be uniquely represented in the form $\cup_{i=1}^{n}\left(a_{i}, b_{i}\right]$, where $n \geqslant 0, a_{1}, b_{1}, \ldots, a_{n}, b_{n} \in J^{+}$, and $-\infty \leqslant a_{1}<b_{1}<a_{2}<\cdots<a_{n}<b_{n} \leqslant \infty$. We call this representation the canonical representation of $a$. We denote $\sigma_{a}=\left\{a_{1}, b_{1}, \ldots, a_{n}, b_{n}\right\}$. Note that $\sigma_{a-b}, \sigma_{a \cap b}, \sigma_{a \cup b} \subseteq \sigma_{a} \cup \sigma_{b}$. Let $\vec{\sigma}_{a}=\left\langle a_{1}, b_{1}, \ldots, a_{n}, b_{n}\right\rangle$. We make the convention that $\operatorname{Dom}\left(\vec{\sigma}_{a}\right)=\{1, \ldots, 2 n\}$.

Note that if $\left\langle J,\langle\rangle\right.$ is a linear ordering and $\varnothing \neq J_{1} \subseteq J$, then $B\left(J_{1}\right)$ can be embedded in $B(J)$ in a natural way; so we regard $B\left(J_{1}\right)$ as a subalgebra of $B(J)$.

The following theorem answers affirmatively a question of B. Rotman [R, Conjecture B]. 
THEOREM 5.1. Every subalgebra of an interval algebra is retractive.

Proof. We prove the following statement which is equivalent to the theorem. If $\langle J,\langle\rangle$ is a linear ordering, $B=B(J)$ is its interval algebra. $I \subseteq B$ is an ideal, and $A \subseteq B$ is a subalgebra of $B$; then there is a subalgebra $A^{\prime} \subseteq A$, such that for every $a \in A\left|A^{\prime} \cap a / I\right|=1$.

Let $J_{0} \subseteq J^{+}$be a maximal set with the property that $B\left(J_{0}\right) \cap A \cap I=\{0\}$. We will show that for every $a \in A,\left|B\left(J_{0}\right) \cap A \cap a / I\right|=1$, so $A^{\prime}$ can be chosen to be $B\left(J_{0}\right) \cap A$.

For every $a \in B$ let $\bar{\sigma}_{a}=\sigma_{a}-J_{0} . \bar{\sigma}_{a}=\varnothing$ iff $a \in B\left(J_{0}\right)$. We will prove if $a \in A$ and $\overline{\boldsymbol{\sigma}}_{a} \neq \varnothing$, then there is $a^{\prime} \in A$ such that $a / I=a^{\prime} / I$ and $\left|\bar{\sigma}_{a^{\prime}}\right|<\left|\bar{\sigma}_{a}\right|$. Let $a \in A$, $a=\cup_{i=1}^{n}\left(a_{i}, b_{i}\right]$ be its canonical representation and suppose $a_{k} \notin J_{0}$. So $B\left(J_{0} \cup\left\{a_{k}\right\}\right) \cap A \cap I \neq\{0\}$. Let $0 \neq b \in B\left(J_{0} \cup\left\{a_{k}\right\}\right) \cap A \cap I$, and $b=$ $\cup_{i=1}^{m}\left(c_{i}, d_{i}\right]$ be its natural representation. For some $1 \leqslant j \leqslant m$ either: (1) $a_{k}=c_{j}$, or (2) $a_{k}=d_{j}$, and $\sigma_{b}-\left\{a_{k}\right\} \subseteq J_{0}$.

If (1) happens, then $\sigma_{a-b} \boxplus a_{k}$; however, $\sigma_{a-b} \subseteq \sigma_{a} \cup \sigma_{b}$. Since $\sigma_{b}-\left\{a_{k}\right\} \subseteq J_{0}$ $\left|\overline{\boldsymbol{\sigma}}_{a-b}\right|<\left|\overline{\boldsymbol{\sigma}}_{a}\right|$. If (2) happens, then $\sigma_{a \cup b} \boxplus a_{k}$, so $\left|\overline{\boldsymbol{\sigma}}_{a \cup b}\right|<\left|\overline{\boldsymbol{\sigma}}_{a}\right|$. Since $b \in I, a / I=a$ $-b / I=a \cup b / I$, and since $b \in A, a-b, a \cup b \in A$. A similar argument holds when for some $k b_{k} \in \bar{\sigma}_{a}$.

So, by descending induction, for every $a \in A$ there is $a^{\prime} \in A \cap B\left(J_{0}\right)$ such that $a / I=a^{\prime} / I$. Since $B\left(J_{0}\right) \cap A \cap I=\{0\}$, for every $a \in A\left|B\left(J_{0}\right) \cap A \cap a / I\right| \leqslant 1$. So $A^{\prime} \stackrel{\text { def }}{=} B\left(J_{0}\right) \cap A$ is as desired. Q.E.D.

Let $B_{1} \times B_{2}$ be the direct product of $B_{1}$ and $B_{2}$. A Boolean term $\tau\left(x_{1}, \ldots, x_{n}\right)$ is called trivial if the value of $\tau$ under every assignment into a BA is 0 . Otherwise $\tau$ is said to be nontrivial.

An $n$-tuple $\left\langle a_{1}, \ldots, a_{n}\right\rangle$ of elements of a BA $B$ is dependent if for some nontrivial $\tau\left(x_{1}, \ldots, x_{n}\right): \tau\left(a_{1}, \ldots, a_{n}\right)=0$.

Let $\left\langle J,\langle\rangle\right.$ be a linear ordering $B=B(J), a \in B$ and $\left|\sigma_{a}\right|=k$. If $\vec{\sigma}_{a}(1)>-\infty$, we define $\vec{\sigma}_{a}(0)=-\infty$; if $\vec{\sigma}_{a}(k)<\infty$, we define $\vec{\sigma}_{a}(k+1)=\infty$. So, $\{1, \ldots, k\} \subseteq$ $\operatorname{Dom}\left(\vec{\sigma}_{a}\right) \subseteq\{0, \ldots, k+1\}$.

Definition 5.2. Let $\left\langle J,\langle\rangle\right.$ be a linear ordering, $B=B(J), \vec{a}=\left\{a_{i} \mid i<\alpha\right\} \subseteq B$. $\vec{a}$ is called homogeneous if: (1) there is $k$ such that for every $i<\alpha\left|\sigma_{a_{i}}\right|=k$; and (2) for every $1<j<\alpha: \sigma_{a_{i}} \cap\{-\infty, \infty\}=\sigma_{a_{j}} \cap\{-\infty, \infty\}, \sigma_{a_{i}} \cap \sigma_{a_{j}}-\{-\infty, \infty\}=\varnothing$, and there is $l=l_{i j}^{\vec{a}}<\max \left(\operatorname{Dom}\left(\vec{\sigma}_{a_{i}}\right)\right), l \in \operatorname{Dom}\left(\vec{\sigma}_{a_{i}}\right)$, such that $\vec{\sigma}_{a_{i}}(l)<\infty$, and $\vec{\sigma}_{a_{i}}(l)<\sigma_{a_{j}}-\{-\infty, \infty\}<\vec{\sigma}_{a_{i}}(l+1)$.

Note that $\vec{a}$ might be the constant sequence of 0 's or the constant sequence of l's.

LEMMA 5.3. There is $n_{0}<\omega$ such that for every homogeneous $\vec{a}$, every sequence of $n_{0}$ elements of $\vec{a}$ is dependent.

Proof. Easy to check.

Lemma 5.4. Let $J$ and $B$ be as in 5.2, and let $P \subseteq B,|P|=\lambda>\aleph_{0}$, and $\lambda$ is regular; then there is a 1-1 sequence $\left\{a_{i} \mid i<\lambda\right\} \subseteq P, n<\omega$ and $-\infty=b_{0}<b_{1}<$ $\cdots<b_{n}=\infty$, such that for every $m<n\left\{a_{i} \cap\left(b_{m}, b_{m+1}\right] \mid i<\lambda\right\}$ is homogeneous in $B \uparrow\left(b_{m}, b_{m+1}\right]$. 
Proof. By a simple cleaning process.

The following theorem is a weakened version of Theorem 2(b) in [Ru] which had an error. At present we do not know to prove or disprove it.

THEOREM 5.5. Let $J, B, P$, and $\lambda$ be as in 5.4. Then there is $m<\omega$ and a 1-1 $\vec{a}=\left\{a_{i} \mid i<\lambda\right\} \subseteq P$, such that every $m$ elements of $\vec{a}$ are dependent.

Proof. Let $n, b_{0}, \ldots, b_{n}$ and $\vec{a}=\left\{a_{i} \mid i<\lambda\right\}$ be as in 5.4 and let $m=n \cdot n_{0} \cdot\left(n_{0}\right.$ was defined in 5.3.)

Let $c_{1}^{1}, \ldots, c_{n_{0}}^{1}, c_{1}^{2}, \ldots, c_{n_{0}}^{2}, \ldots, c_{1}^{n}, \ldots, c_{n_{0}}^{n}$ be elements of $\vec{a}$. By 5.3 for every $i<n$, there is a nontrivial term $\tau_{i}\left(x_{1}, \ldots, x_{n_{0}}\right)$, such that in $B \uparrow\left(b_{i}, b_{i+1}\right]$ :

$$
\tau_{i}\left(c_{1}^{i} \cap\left(b_{i}, b_{i+1}\right], \ldots, c_{n_{0}}^{i} \cap\left(b_{i}, b_{i+1}\right]\right)=0 .
$$

Let $\tau\left(x_{1}^{1}, \ldots, x_{n_{0}}^{1}, \ldots, x_{1}^{n}, \ldots, x_{n_{0}}^{n}\right)=\bigcap_{i=1}^{n} \tau_{i}\left(x_{1}^{i}, \ldots, x_{n_{0}}^{i}\right)$; then $\tau$ is nontrivial and

$$
\tau\left(c_{1}^{1}, \ldots, c_{n_{0}}^{1}, \ldots, c_{1}^{n}, \ldots, c_{n_{0}}^{n}\right)=0 \text {. Q.E.D. }
$$

An object $C=\left\langle\left\{a_{i} \mid i<\lambda\right\},\left\langle b_{0}, \ldots, b_{n}\right\rangle\right\rangle$ which satisfies the conclusion of 5.4 is called a system.

If $\vec{a}=\left\{a_{i} \mid i<\lambda\right\} \subseteq B$ is homogeneous, let $m(\vec{a}, B)=\left|\sigma\left(a_{i}\right)-\{-\infty, \infty\}\right|$, where $i$ is any ordinal $<\lambda$. If $C$ is as above, we define

$$
\begin{aligned}
m(C)= & 2 \cdot \sum_{j<n} m\left(\left\{a_{i} \cap\left(b_{j}, b_{j+1}\right] \mid i<\lambda\right\}, B \uparrow\left(b_{j}, b_{j+1}\right]\right) \\
& -\mid\left\{j \mid\left\{a_{i} \cap\left(b_{j}, b_{j+1}\right] \mid i<\lambda\right\} \text { is not a constant sequence }\right\} \mid .
\end{aligned}
$$

Let us now quote Theorem 3 from [BaK].

If $\lambda$ is a regular cardinal and $B$ does not contain an antichain of power $\lambda$, then $B$ contains a dense subset of power $<\lambda$.

THEOREM 5.6. Let $\langle J,\langle\rangle$ be a linear ordering, and let $B \subseteq B(J)$ be of power $\lambda$, where $\lambda$ is regular. Then $B$ contains a chain or an antichain of power $\lambda$.

Proof. Suppose $|B|=\lambda$, and $B$ does not contain antichains of power $\lambda$. Let $C=\left\langle\vec{a},\left\langle b_{0}, \ldots, b_{n}\right\rangle\right\rangle$ be a system, where $\vec{a}$ is $1-1$ and has length $\lambda$, and $m(C)$ is minimal. We will show that for some $D \subseteq \lambda,|D|=\lambda$ and $\left\{a_{\alpha} \mid \alpha \in D\right\}$ is a chain. Let $\vec{a}=\left\{a_{j} \mid j<\lambda\right\}$. For every $i<n$, let $a_{j}^{i}=a_{j} \cap\left(b_{i}, b_{i+1}\right], \vec{a}^{i}=\left\{a_{j}^{i} \mid j<\lambda\right\}$, and $B^{i}=B \nmid\left(b_{i}, b_{i+1}\right]$. Note that since $B$ does not have antichains of power $\lambda$, neither does $B^{i}$. We shall first show that it can be assumed that for every $i<n$ :

(*) for every $j<k<\lambda, a_{j}^{i}$ and $a_{k}^{i}$ are comparable.

Let $m_{i}=\left|\sigma_{a_{j}}-\{-\infty, \infty\}\right| .(*)$ certainly holds for $i$, if $\left|m_{i}\right| \leqslant 1$. Suppose $m_{i}>1$. By duality, we can w.l.o.g. assume that $-\infty \notin \sigma_{a_{i}^{i}}$. Let $D_{i}=\{j \mid$ there is $k>j$ such that $\left.1 \leqslant l_{j k}^{\vec{a}^{i}} \leqslant m_{i}-1\right\}$. ( $l_{j k}^{\vec{a}}$ was defined in 5.2.) Suppose $\left|D_{i}\right|=\lambda$, for every $j \in D_{i}$, let $k(j)$ be as assured in the definition of $D_{i}$. For every $j \in D_{i}$, let $c_{j}=a_{j}^{i}-a_{k(j)}^{i}$. Let $E \subseteq B^{i}-\{0\}$ be dense in $B^{i}$ and $|E|<\lambda$. Since for every $j \in D_{i}, c_{j} \neq 0$, there is $e_{j} \in E$ such that $e_{j} \subseteq c_{j}$. Since $\lambda$ is regular, there is $e \in E$ such that: $D^{\prime} \stackrel{\text { def }}{=}\left\{j \mid e_{j}=e\right\}$ has power $\lambda$. Let $t=\min \left(\sigma_{e}\right)$; then for every $j \in D^{\prime}, \vec{\sigma}_{a_{j}^{j}}(1)<t<\vec{\sigma}_{a_{j}^{i}}\left(m_{i}\right)$. But then 
there is $l, 1 \leqslant l<m_{i}$ such that $D^{\prime \prime} \stackrel{\text { def }}{=}\left\{j \mid \vec{\sigma}_{a^{\prime}}(l)<t<\vec{\sigma}_{a_{j}^{\prime}}(l+1)\right\}$ has power $\lambda$. Let

$$
C^{\prime}=\left\langle\left\{a_{j} \mid j \in D^{\prime \prime}\right\},\left\langle b_{0}, \ldots, b_{i}, t, b_{i+1}, \ldots, b_{n}\right\rangle\right\rangle ;
$$

then $m\left(C^{\prime}\right)=m(C)-1$, a contradiction.

So $\left|D_{i}\right|<\lambda$. By deleting $\left\{a_{j} \mid j \in \cup\left\{D_{i} \mid m_{i}>1\right\}\right\}$ from $\left\{a_{j} \mid j<\lambda\right\}$, we can assume that for every $i<n$ if $m_{i}>1$, then $D_{i}=\varnothing$.

It is easy to check that if $D_{i}=\varnothing$, then either: for every $j<k<\lambda, a_{j}^{i}$ and $a_{k}^{i}$ are comparable; or for every $j<k<\lambda a_{j}^{i}$ and $a_{k}^{i}$ are incomparable. Since the second case cannot hold, $(*)$ is proved. For every $i, j<n$ and for every $k<\lambda$, let $D_{i j k}=\left\{\alpha \mid \alpha<\lambda, a_{\alpha}^{i} \subsetneq a_{k}^{i}\right.$ and $\left.a_{k}^{j} \subsetneq a_{\alpha}^{j}\right\}$. We prove that for every $i, j, k$ as above, $\left|D_{i j k}\right|<\lambda$. If not, let $i, j, k$ be a counterexample. For every $\alpha \in D_{i j k}$ let $b^{\alpha}=a_{\alpha} \cap$ $a_{k}$, so $b^{\alpha} \cap\left(b_{j}, b_{j+1}\right]=a_{k}^{j}$, and $b^{\alpha} \cap\left(b_{i}, b_{i+1}\right]=a_{\alpha}^{i}$. Thus $\left\{b^{\alpha} \mid \alpha \in D_{i j k}\right\}$ is 1-1. Let $\vec{c}$ be a subsequence of length $\lambda$ of $\left\{b^{\alpha} \mid \alpha \in D_{i j k}\right\}$, such that for some $b_{0}^{\prime}, \ldots, b_{n^{\prime}}^{\prime}$, $C^{\prime} \stackrel{\text { def }}{=}\left\langle\vec{c},\left\langle b_{0}^{\prime}, \ldots, b_{n^{\prime}}^{\prime}\right\rangle\right\rangle$ is a system; then $m\left(C^{\prime}\right)<m(C)$, a contradiction, so $\left|D_{i j k}\right|<$ $\lambda$.

We now define by induction $D \subseteq \lambda,|D|=\lambda$, such that $\left\{a_{\alpha} \mid \alpha \in D\right\}$ is a chain. Suppose $\alpha_{\nu}$ have been defined for every $\nu<\xi$. Let $\alpha_{\xi} \in \lambda-\cup\left\{D_{i j \nu} \mid i, j<n\right.$ and $\nu<\xi\}-\left\{\alpha_{\nu} \mid \nu<\xi\right\}$. Let $D=\left\{\alpha_{\nu} \mid \nu<\lambda\right\}$; then $\left\{a_{\alpha} \mid \alpha \in D\right\}$ is a chain. Q.E.D.

COROLlaRy 5.7. If $B$ is $S C$, then every subalgebra of $B$ which is embeddable in an interval algebra has power $\leqslant \boldsymbol{\aleph}_{0}$.

Proof. $B$ does not contain uncountable chains or antichains, so the corollary follows from Theorem 5.6. (Alternatively 5.5 can be used.) ${ }^{\prime}$

Our last goal in this section is to prove, assuming $\mathrm{CH}$, that the theory of BA's in Magidor - Malitz language $L^{2}$ is undecidable. (See definition in [MM].)

Sierpinski assuming $\mathrm{CH}$ (but see also Bonnet [B1]) constructed a family $\left\{L_{\alpha} \mid \alpha<\right.$ $2^{\aleph_{1}}$ \} of subsets of $\mathbf{R}$, such that if $f$ is an order preserving or order reversing function, and $\operatorname{Dom}(f) \subseteq L_{\alpha}, \operatorname{Rng}(f) \subseteq L_{\beta}$ and $\alpha \neq \beta$, then $|\operatorname{Dom}(f)| \leqslant \boldsymbol{\aleph}_{0}$. It is easy to see that if $\alpha \neq \beta$, then every linear ordering which is embeddable in both $B\left(L_{\alpha}\right)$ and $B\left(L_{\beta}\right)$ is of power $\leqslant \boldsymbol{N}_{0}$.

We now show how to interpret in the $L^{2}$-theory of BA's the first order theory of symmetric irreflexive relations. Let $h:\left\{\langle\sigma, \alpha\rangle|\sigma \subseteq \omega,| \sigma \mid=2, \alpha<\aleph_{1}\right\} \rightarrow \aleph_{1}$ be a 1-1 function. Let $B_{\sigma, \alpha}=B\left(L_{h(\sigma, \alpha)}\right)$, where $\left\{L_{\alpha} \mid \alpha<2^{\kappa_{1}}\right\}$ is as mentioned above.

Let $M=\langle\beta, R\rangle$ be a structure, such that $\beta \leqslant \omega$ and $R$ is an irreflexive symmetric relation on $\alpha$.

We now define a BA $B_{M}$, in which $M$ can be interpreted. For every $i \in \beta$, let $B_{i}=\sum\left\{B_{\{i, j\}, \alpha} \mid\langle i, j\rangle \in R, \alpha<\boldsymbol{N}_{1}\right\}$ where $\sum$ denotes the weakest direct product. Let $B_{M}=\sum_{i \in \beta} B_{i}$. Let $\varphi_{0}(x)$ be the formula in $L^{2}$ that says: "there is an uncountable family of pairwise disjoint nonzero subelements of $x$." Let

$$
\varphi_{1}(x) \equiv \varphi_{0}(x) \wedge \forall y_{1}, y_{2}\left(\left[\left(y_{1} \cup y_{2}=x\right) \wedge\left(y_{1} \cap y_{2}=0\right)\right] \rightarrow \bigvee_{i=1}^{2} \neg \varphi_{0}\left(y_{i}\right)\right) .
$$

Let $\varphi_{2}(x, y)=\varphi_{1}(x) \wedge \varphi_{1}(y) \wedge \neg \varphi_{0}(x \Delta y)$. It is clear that in $B_{M} \varphi_{2}(x, y)$ defines an equivalence relation $E$ on $\left\{a \mid B_{M} \vDash \varphi_{1}[a]\right\}$, and for every equivalence class $C$ of 
$E$, there is a unique $i<\beta$ such that $1_{B_{i}} \in C$. Let us denote this equivalence class by $C_{i}$.

Let $\varphi_{3}\left(y_{1}, y_{2}\right)$ be the formula which says:

$(\exists X)\left(|X| \geqslant \aleph_{1} \wedge\left(\forall x_{1} x_{2} \in X\right)\left(\left(x_{1}\right.\right.\right.$ and $x_{2}$ are comparable $)$

$$
\wedge\left[\left(x_{1}=x_{2}\right) \vee\left(\left(x_{1} \cap y_{1} \neq x_{2} \cap y_{1} \wedge\left(x_{1} \cap y_{2} \neq x_{2} \cap y_{2}\right)\right)\right]\right) .
$$

$\varphi_{3}\left(y_{1}, y_{2}\right)$ says that there is an uncountable linear ordering which is embeddable in both $B \uparrow y_{1}$ and in $B \uparrow y_{2}$.

Let $\varphi_{4}\left(x_{1}, x_{2}\right)$ be the formula that says: $(\exists X)\left(|X| \geqslant \boldsymbol{\aleph}_{1} \wedge\left(\forall y_{1} y_{2} \in X\right)\left(y_{1}=y_{2} \vee\right.\right.$ $\left.\left.\left(y_{1} \cap y_{2}=0 \wedge \varphi_{3}\left(y_{1} \cap x_{1}, y_{1} \cap x_{2}\right)\right)\right)\right)$.

Then, for every $i, j \in \beta$ and for every $x_{1} \in C_{i}$ and $x_{2} \in C_{j}: B_{M} \vDash \varphi_{4}\left[x_{1}, x_{2}\right]$ iff $\langle i, j\rangle \in R$.

Conclusion 5.8. $(\mathrm{CH})$ The first order theory of irreflexive symmetric relations is interpretable in the $L^{2}$-theory of BA's, and so the $L^{2}$-theory of BA's is undecidable.

6. Retractiveness of free products. We have already seen that $\nabla_{\kappa_{1}}$ implies the existence of a retractive BA not embeddable in an interval algebra. The results of this section are motivated by the following open question.

Question 6.1. Does ZFC imply the existence of a retractive BA not embeddable in an interval algebra?

Throughout this section $B_{1} * B_{2}$ denotes the free product of $B_{1}$ and $B_{2}$ and $\tilde{B}$ denotes the BA of finite and cofinite subsets of $\omega$. Rotman $[\mathbf{R}]$ has proved that if $B_{1}$ is infinite and $B_{2}$ is uncountable, then $B_{1} * B_{2}$ is not embeddable in an interval algebra.

We shall show that if $L$ is a Suslin ordering, or if $L$ is a Sierpinski set then $B(L) * \tilde{B}$ is retractive. In fact in Theorem 6.6 we shall find a necessary and sufficient condition on $L$ that assures that $B(L) * \tilde{B}$ is retractive. We shall conclude that if there is a Suslin tree, or if MA holds, then there is a retractive BA not embeddable in an interval algebra.

In this section $B$ is considered to be an ideal in itself; an ideal $I \neq B$ is called a proper ideal. If $I$ is an ideal in $B$ and $A$ is a subalgebra of $B$ we say that $A$ is a retract of $B$ relative to $I$ if, for every $b \in B,|b / I \cap A|=1$; an endomorphism $h$ of $B$ with kernel $I$ such that $h^{2}=h$ is called a retraction of $B$ relative to $I$. Note that if $h$ is a retraction of $B$ relative to $I$, then $\operatorname{Rng}(h)$ is a retract of $B$ relative to $I$. If $I$ is an ideal in $B$ and $a \in B$ let $I \uparrow a=\{b \mid b \subseteq a$ and $b \in I\}$; clearly $I \uparrow a$ is an ideal in $B \nmid a$.

LEMMA 6.2. (a) Let $\mathrm{P}_{1}$ denote the following property of a $B A B$; (i) $B$ is retractive; and (ii) for every sequence $\left\{I_{i} \mid i \in \omega\right\}$ of ideals in $B$, there is a sequence $\left\{\left\langle A_{i}, a_{i}\right.\right.$, $\left.\left.h_{i}\right\rangle \mid i \in \omega\right\}$ such that (1) $\left\{A_{i} \mid i \in \omega\right\}$ is an increasing sequence of subalgebras of $B$ whose union is $B$; (2) $a_{i} \in A_{i} \cap I_{i}$; and (3) $h_{i}$ is a retract of $B \uparrow-a_{i}$ relative to $I_{i} \uparrow-a_{i}$, and $h_{i} \uparrow\left(A_{i} \uparrow-a_{i}\right)=\mathrm{Id}$.

If $B$ has property $\mathrm{P}_{1}$ then $B * \tilde{B}$ is retractive.

(b) Let $\mathrm{P}_{2}$ be the following property of a $B A B$ : for every sequence $\left\{I_{i} \mid i \in \omega\right\}$ of ideals in $B$ there is an increasing sequence $\left\{A_{j} \mid j \in \omega\right\}$ of subalgebras of $B$ whose union is $B$, and for every $i, j \in \omega$ there is $a_{i j} \in I_{i}$ such that for every $b \in I_{i} \cap A_{j} ; b \subseteq a_{i j}$.

If for some infinite $B_{1} B * B_{1}$ is retractive, then $\mathrm{P}_{2}$ holds in $B$. 
Proof. (a) Let $\tilde{B}$ have property $\mathrm{P}_{1}$ and let $B=\bar{B} * \tilde{B}$. We denote by $1, \overline{1}$ and $\tilde{\mathrm{l}}$ the ones of $B, \bar{B}$ and $\tilde{B}$ respectively. Let $\left\{e_{i} \mid i \in \omega\right\}$ be a 1-1 enumeration of the atoms of $\tilde{B}$.

Let $I$ be an ideal in $B$; we define the following ideals in $\bar{B}: I_{i}=\left\{b \in \bar{B} \mid b \cap e_{i} \in\right.$ $I\}, J=\left\{b \in \bar{B} \mid\right.$ for some $\left.n \in \omega b \cap\left(\tilde{1}-\cup_{i<n} e_{i}\right) \in I\right\}$. Let $\left\{\left\langle A_{i}, a_{i}, h_{i}\right\rangle \mid i \in \omega\right\}$ be as assured by $\mathrm{P}_{1}$ for the sequence of ideals $\left\{I_{i} \mid i \in \omega\right\}$. If $J=\bar{B}$, then by the retractiveness of $\bar{B}$ it is easy to find a retract of $B$ relative to $I$. We thus assume that $J \neq \bar{B}$. Let $C$ be a retract of $\bar{B}$ relative to $J$.

Let $g_{i}$ be the endomorphism of $\bar{B}$ extending Id $\left.|\bar{B}| a_{i}\right) \cup h_{i}$. For every $b \in \bar{B}$ let $b^{*}=\cup_{i \in \omega}\left(g_{i}(b) \cap e_{i}\right)$. A priori it is clear that $*$ is a homomorphism of $\bar{B}$ into the completion of $B$, but since $\left\{A_{i} \mid i \in \omega\right\}$ is an increasing sequence of subalgebras of $\bar{B}$ whose union in $B$, and by the definition of the $g_{i}$ 's, * is really an embedding of $\bar{B}$ into $B$. Let $C^{*}=\left\{c^{*} \mid c \in C\right\}$, and let $C_{i}=\left\{b \cap e_{i} \mid b \in \operatorname{Rng}\left(h_{i}\right)\right\}$. Let $A$ be the subalgebra of $B$ generated by $C^{*} \cup \cup_{i \in \omega} C_{i}$. We claim that $A$ is a retract of $B$ relative to $I$.

We first show that for every $b \in B|b / I \cap A| \geqslant 1$. Let $b \in B$; then for some $b_{1} \in \bar{B}$ and $n \in \omega$

$$
b \cap\left(\bar{l} \cap\left(\tilde{\mathrm{l}}-\bigcup_{i<n} e_{i}\right)\right)=b_{1} \cap\left(\tilde{\mathrm{l}}-\bigcup_{i<n} e_{i}\right) .
$$

Let $c \in C$ be such that $c \triangle b_{1} \in J$. Let $m$ be chosen so that: $m \geqslant n$,

$$
c^{*} \cap\left(\overline{\mathrm{l}} \cap\left(\tilde{\mathrm{l}}-\bigcup_{i<m} e_{i}\right)\right)=c \cap\left(\tilde{\mathrm{l}}-\bigcup_{i<m} e_{i}\right)
$$

and

$$
\left(c \triangle b_{1}\right) \cap\left(\tilde{\mathrm{l}}-\bigcup_{i<m} e_{i}\right) \in I .
$$

Let $d=\left(c^{*} \triangle b\right) \cap\left(\overline{1} \cap \cup_{i<m} e_{i}\right) ;$ so for some $d_{0}, \ldots, d_{m-1} \in \bar{B}, \quad d=$ $\cup_{i<m}\left(d_{i} \cap e_{i}\right)$. Let $d_{i}^{\prime}=h_{i}\left(d_{i}-a_{i}\right)$ and let $d^{\prime}=\cup_{i<m}\left(d_{i}^{\prime} \cap e_{i}\right)$. It is easy to check that $d \triangle d^{\prime} \in I$.

Clearly $d^{\prime} \triangle c^{*} \in A$, and we show that $\left(d^{\prime} \triangle c^{*}\right) \triangle b \in I$.

$$
\begin{aligned}
\left(d^{\prime} \triangle c^{*}\right) \triangle b= & \left(\left(d^{\prime} \triangle c^{*} \triangle b\right) \cap\left(\overline{1} \cap \bigcup_{i<m} e_{i}\right)\right) \\
& \cup\left(\left(d^{\prime} \triangle c^{*} \triangle b\right) \cap\left(\overline{1} \cap\left(\tilde{1}-\bigcup_{1<m} e_{i}\right)\right)\right) \\
= & \left(d^{\prime} \triangle d\right) \cup\left(c^{*} \triangle b\right) \in I .
\end{aligned}
$$

We have thus proved that for every $b \in B|b / I \cap A| \geqslant 1$.

We now prove that $A \cap I=\{0\}$. It is easy to see that every element of $A$ is a finite union of elements of the following forms: (1) $a \cap b$, where $a \in C^{*}$ and for some $i b \in C_{i}$; (2) $a-\cup_{j=1}^{k} c_{j}$, where $a \in C^{*}$, and there are $i_{1}, \ldots, i_{k}$ such that $c_{1} \in C_{i_{1}}, \ldots, c_{k} \in C_{i_{k}}$. It suffices to show that every element of one of the above forms that belongs to $I$ is equal to 0 . 
Suppose $a \in C^{*}, b \in C_{i}$ and $a \cap b \in I$. Hence there is $b^{\prime} \in \operatorname{Rng}\left(h_{i}\right)$ such that $b=b^{\prime} \cap e_{i}$, and for some $a^{\prime} \in \operatorname{Rng}\left(g_{i}\right): a \cap\left(\overline{1} \cap e_{i}\right)=a^{\prime} \cap e_{i}$. By the definition of $g_{i} a^{\prime} \cap b^{\prime} \in \operatorname{Rng}\left(h_{i}\right) . a \cap b=\left(a^{\prime} \cap b^{\prime}\right) \cap e_{i}$, so by the definition of $I_{i} a^{\prime} \cap b^{\prime} \in$ $I_{i}$. But $\operatorname{Rng}\left(h_{i}\right) \cap I_{i}=\{0\}$, hence $a^{\prime} \cap b^{\prime}=0$, and hence $a \cap b=0$.

Let $a \in C^{*}, c_{j} \in C_{i}, j=1, \ldots, k$, and $a-\cup_{j=1}^{k} c_{j} \in I$. Let $d \in \bar{B}$ be such that $a=d^{*}$, hence $d \in C$. Let $n>i_{1}, \ldots, i_{k}$ be such that

$$
a \cap\left(\bar{l} \cap\left(\tilde{\mathrm{l}}-\bigcup_{i<n} e_{i}\right)\right)=d \cap\left(\tilde{\mathrm{l}}-\bigcup_{i<n} e_{i}\right)
$$

hence

$$
\left(a-\bigcup_{j=1}^{h} c_{j}\right) \cap\left(\overline{\mathrm{l}} \cap\left(\tilde{\mathrm{l}}-\bigcup_{i<n} e_{i}\right)\right)=d \cap\left(\tilde{\mathrm{l}}-\bigcup_{i<n} e_{i}\right) .
$$

This means that $d \in J$. But $J \cap C=\{0\}$; hence $d=0$, hence $d^{*}=0$. Q.E.D.

(b) Suppose $B_{1}$ is infinite, and $\bar{B} * B_{1}$ is retractive; we show that $\mathrm{P}_{2}$ holds in $\bar{B}$. Let $\left\{I_{i} \mid i \in \omega\right\}$ be a sequence of ideals in $\bar{B}$. Let $\left\{J_{j} \mid j \in \omega\right\}$ be an enumeration of $\left\{I_{i} \mid i \in \omega\right\}$ such that for every $i \in \omega\left\{j \mid J_{j}=I_{i}\right\}$ is infinite. Let $\tilde{1}$ denote $1_{B_{1}}$, and let $\left\{e_{i} \mid i \in \omega\right\}$ be a sequence of nonzero pairwise disjoint elements of $B_{1}$. Let $\tilde{B}$ be the subalgebra of $B_{1}$ generated by $\left\{e_{i} \mid i \in \omega\right\}$, and let $I$ be the ideal in $B$ generated by $\cup_{j \in \omega}\left\{b \cap e_{j} \mid b \in J_{j}\right\}$. Let $h$ be a retraction of $B$ relative to $I$. Let

$$
A_{j}=\left\{b \in \bar{B} \mid h(b \cap \tilde{1}) \cap\left(\overline{1} \cap\left(\tilde{1}-\bigcup_{k<j} e_{k}\right)\right)=b \cap\left(\tilde{1}-\bigcup_{k<j} e_{k}\right)\right\} .
$$

It is easy to see that $\left\{A_{j} \mid j \in \omega\right\}$ is an increasing sequence of subalgebras whose union is $\bar{B}$.

Let $i, j \in \omega$. There is $k \geqslant j$ such that $J_{k}=I_{i}$. Let $a_{i j} \in \bar{B}$ be such that $h\left(\overline{1} \cap e_{k}\right)$ $\cap\left(\overline{1} \cap e_{k}\right)=-a_{i j} \cap e_{k}$. Clearly $a_{i j} \in J_{k}=I_{i}$.

Let $a \in A_{j} \cap I_{i}$, then $a \in A_{k} \cap I_{k}$, hence $a \cap e_{k} \in I$, so

$$
\begin{aligned}
0 & =h\left(a \cap e_{k}\right)=h(a \cap \tilde{1}) \cap h\left(\overline{1} \cap e_{k}\right) \\
& \supseteq\left(a \cap e_{k}\right) \cap\left(-a_{i j} \cap e_{k}\right)=\left(a \cap-a_{i j}\right) \cap e_{k} .
\end{aligned}
$$

So $a \cap-a_{i j}=0 . a \subseteq a_{i j}$. Q.E.D.

The following lemma follows from the work of E. van Douwen [D1]. We bring it here for the sake of completeness.

LEMMA 6.3. If $\bar{B}$ contains a nonprincipal noncountably generated ideal, and $B^{\prime}$ is infinite, then $\bar{B} * B^{\prime}$ is not retractive.

Proof. Let $B=\bar{B} * B^{\prime}$, and let $1, \overline{1}$ and $1^{\prime}$ denote the ones of $B, \bar{B}, B^{\prime}$ respectively. Let $\left\{b_{i} \mid i<\boldsymbol{\aleph}_{1}\right\}$ be a sequence of elements of $\bar{B}$ such that for every $n \in \omega$ and $j_{1}, \ldots, j_{n}<j<\boldsymbol{\aleph}_{1} b_{j} \not \cup_{m=1}^{n} b_{j_{m}}$. Let $\left\{a_{k} \mid k \in \omega\right\}$ be a strictly increasing sequence of elements of $B^{\prime}$, and let $I$ be the ideal in $B$ generated by $\left\{b_{j} \cap a_{k} \mid j<\aleph_{1}, k<\omega\right\}$. Suppose by contradiction there is a retraction $c \mapsto c^{*}$ of $B$ relative to $I$. 
For every $i<\aleph_{1}$ there is $k_{i}<\omega$ such that $\left(b_{i} \cap 1^{\prime}\right)^{*} \supseteq b_{i} \cap\left(1^{\prime}-a_{k_{1}}\right)$. Let $k$ be such that $D \stackrel{\text { def }}{=}\left\{i \mid k_{i}=k\right\}$ is uncountable. Let $m>k$; then there are $i_{1}, \ldots, i_{n}<\boldsymbol{\aleph}_{1}$ such that $\left(\overline{1} \cap a_{m}\right)^{*} \supseteq\left(\overline{1}-\cup_{l=1}^{n} b_{i_{1}}\right) \cap a_{m}$. Let $i>i_{1}, \ldots, i_{n}$; then

$$
\begin{aligned}
0 & =\left(b_{i} \cap a_{m}\right)^{*}=\left(b_{i} \cap 1^{\prime}\right)^{*} \cap\left(\overline{1} \cap a_{m}\right)^{*} \\
& \supseteq\left(b_{i} \cap\left(1^{\prime}-a_{k}\right)\right) \cap\left(\left(\overline{1}-\bigcup_{l=1}^{n} b_{i_{l}}\right) \cap a_{m}\right) \\
& =\left(b_{i}-\bigcup_{l=1}^{n} b_{i_{l}}\right) \cap\left(a_{m}-a_{k}\right) \neq 0 .
\end{aligned}
$$

A contradiction, so the lemma is proved. Q.E.D.

Definition 6.4. (a) Let $L$ be a linear ordering. An open interval tree (OIT) for $L$ is a sequence $G=\left\{G_{i} \mid i \in \omega\right\}$ such that each $G_{i}$ is a family $\left\{G_{i j} \mid j \in \alpha_{i}\right\}$ of pairwise disjoint open convex sets, and for every $i<k<\omega$ and $j \in \alpha_{k}$ there is $m \in \alpha_{i}$ such that $G_{k j} \subseteq G_{i m}$.

(b) Let $G$ be an OIT for $L$ and $A \subseteq L$. $A$ is called $G$-small if for every $i \in \omega$ $\left\{j \in \alpha_{i} \mid A \cap G_{i j} \neq \varnothing\right\}$ is finite. $A$ is called $\sigma-G$-small if $A$ is a countable union of $G$-small sets.

(c) $L$ is thin if: (1) $\mid\{a \in L \mid a$ has a successor in $L\} \mid \leqslant \boldsymbol{\aleph}_{0}$; and (2) for every OIT $G, L$ is a $\sigma-G$-small.

Proposition 6.5. If $L$ is thin, then (a) $L$ is c.c.c., i.e. it does not contain an uncountable set of pairwise disjoint open sets; (b) every subset of $L$ is thin; and (c) $L$ is totally disconnected in its order topology.

We leave the easy proof to the reader.

THEOREM 6.6. $B(L) * \tilde{B}$ is retractive iff $L$ is thin. If $L$ is not thin then for every infinite $B^{\prime} B(L) * B^{\prime}$ is not retractive.

Proof. Suppose $L$ is not thin. If $L$ contains an uncountable set of elements which have a successor in $L$, then $B(L)$ has uncountably many atoms, and so the ideal of $B(L)$ generated by its atoms is not countably generated and so by 6.3 for every infinite $B^{\prime} B(L) * B^{\prime}$ is not retractive.

Suppose there is an OIT $G$ such that $L$ is not $\sigma-G$-small. Let $G=\left\{G_{i} \mid i \in \omega\right\}$ and $G_{i}=\left\{G_{i j} \mid j<\alpha_{i} \leqslant \omega\right\}$. We define ideals $\left\{I_{i} \mid i \in \omega\right\} . I_{i}$ is the ideal in $B(L)$ generated by $\left\{(a, b] \mid\right.$ there is $j<\alpha_{i}$ such that $\left.a, b \in G_{i j}\right\}$. Let $\left\{A_{j} \mid j \in \omega\right\}$ be an increasing sequence of subalgebras of $B(L)$ whose union is $B(L)$, and let $L_{j}=$ $\left\{b \mid(-\infty, b] \in A_{j}\right\}$. Clearly $\cup_{j \in \omega} L_{j}=L$. We show that for some $i$ and $j:\{k \mid$ $\left.\left|L_{j} \cap G_{i k}\right| \leqslant 2\right\}$ is infinite. If not, let

$$
L_{j}^{\prime}=L_{j}-\cup\left\{L_{j}-G_{i k} \mid i \in \omega, k \in \alpha_{i} \text { and }\left|L_{j} \cap G_{i k}\right|=1\right\} .
$$

Clearly $L_{j}^{\prime}$ is $G$-small and $\left|L_{j}-L_{j}^{\prime}\right| \leqslant \boldsymbol{\aleph}_{0}$. Hence $\left|L-\cup_{j \in \omega} L_{j}^{\prime}\right| \leqslant \boldsymbol{\aleph}_{0}$. Let $\left\{a_{i} \mid i \in\right.$ $\omega$ \} be an enumeration of $L-\cup_{j \in \omega} L_{j}^{\prime}$, and let $L_{j}^{\prime \prime}=L_{j}^{\prime} \cup\left\{a_{j}\right\}$; then $L_{j}^{\prime \prime}$ is $G$-small and $\cup_{j \in \omega} L_{j}^{\prime \prime}=L$, hence $L$ is $\sigma-G$-small, a contradiction. 
Let $i, j$ be such that $\left\{k|| L_{j} \cap G_{i k} \mid \geqslant 2\right\}$ is infinite. We show that there is no $a \in I_{i}$ such that for every $b \in I_{i} \cap A_{j} b \subseteq a$. Let $a \in I_{i}$; then there are $k_{1}, \ldots, k_{m}<$ $\alpha_{i}, c_{l}, d_{l} \in G_{i k}, l=1, \ldots, m$, such that $a=\cup_{l=1}^{m}\left(c_{l}, d_{l}\right]$. Let $k \neq k_{1}, \ldots, k_{m} c, d \in$ $G_{i k} \cap L_{j}$ and $c<d$. Then $(c, d] \nsubseteq a$ and $(c, d] \in A_{j} \cap I_{i}$. We have thus shown that $B(L)$ does not have property $\mathrm{P}_{2}$, and hence $B(L) * B$ is not retractive unless $B$ is finite.

We now assume that $L$ is thin and prove that $B(L)$ has property $\mathrm{P}_{1}$. In order to avoid some inessential technicalities, we restrict ourselves to the case when $L$ is a dense linear ordering without endpoints; the proof for the general case does not involve additional ideas. Let $\left\{I_{i} \mid i \in \omega\right\}$ be a sequence of ideals in $B(L)$. For every $i \in \omega$ let $\left\{C_{i j} \mid j<\alpha_{i}\right\}$ be the set of convex subsets of $L \cup\{-\infty, \infty\}$ that determines $I_{i}$, that is: for every $j<\alpha_{i}\left|C_{i j}\right|>1$, the $C_{i j}$ 's are pairwise disjoint and $I_{i}$ is generated by $\cup_{j<\alpha_{i}}\left\{(a, b] \mid a, b \in C_{i j}\right\}$. Since $L$ is c.c.c., for every $i, \alpha_{i}$ is countable, and so we assume that $\alpha_{i} \leqslant \omega$. Let $H_{i j}$ be the open set obtained from $C_{i j}$ by deleting its endpoints if they exist. Let $\mathbf{Z}$ denote the set of integers. For every $i, j$ let $\left\{H_{i j z} \mid z \in \mathbf{Z}\right\}$ be a sequence of nonempty pairwise disjoint convex open sets whose union is $H_{i j}$, and such that if $z_{1}<z_{2}$, then $H_{i j z_{1}}<H_{i j z_{2}}$. The existence of such a sequence follows from the total disconnectedness and the c.c.c.-ness of $L$. It is easy to find an OIT $G=\left\{G_{i} \mid i \in \omega\right\}, G_{i}=\left\{G_{i k} \mid k \in \omega\right\}$ such that for every $i, j, z H_{i j z}$ is a union of members of $G_{i}$.

Let $A=\left\{a \mid\right.$ there exist $i, j$ such that $a=\min \left(C_{i j}\right)$ or $\left.a=\max \left(C_{i j}\right)\right\}$, and let $\left\{a_{i} \mid i \in \omega\right\}$ be an enumeration of $A$. Let $\left\{L_{i}^{\prime} \mid i \in \omega\right\}$ be an increasing sequence of $G$-small sets whose union is $(L \cup\{-\infty, \infty\})-A$. It is easy to see that for every $i, j, k, L_{k}^{\prime}$ is bounded in $H_{i j}$, and by the $G$-smallness of $L_{k}^{\prime}$ for every $i$ and $k$ $\left\{j \mid H_{i j} \cap L_{k}^{\prime} \neq \varnothing\right\}$ is finite. So there are finite subsets of $L,\left\{\sigma_{i} \mid i \in \omega\right\}$, such that for every $i$ and $j$, if $\left(L_{i}^{\prime} \cup \cup_{l \leqslant i} \sigma_{l}\right) \cap H_{i j} \neq \varnothing$, then $\left(L_{i}^{\prime} \cup \cup_{l \leqslant i} \sigma_{l}\right) \cap H_{i j}$ has a minimum and a maximum. Let $L_{i}=L_{i}^{\prime} \cup \cup_{l \leqslant i} \sigma_{l} \cup\left\{a_{l} \mid l \leqslant i\right\}$. The sequence $\left\{L_{i} \mid i\right.$ $\in \omega\}$ is thus an increasing sequence whose union is $L \cup\{-\infty, \infty\}$, and for every $i$ : $\left\{j \mid L_{i} \cap C_{i j} \neq \varnothing\right\}$ is finite, and if $L_{i} \cap C_{i j} \neq \varnothing$, then $L_{i} \cap C_{i j}$ has a minimum and a maximum.

Let $A_{i}$ be the subalgebra of $B(L)$ generated by $\left\{(c, d] \mid c, d \in L_{i}\right\}$. Clearly $\left\{A_{i} \mid i \in \omega\right\}$ is an increasing sequence whose union is $B(L)$. Let

$$
b_{i}=\bigcup\left\{\left[\min \left(L_{i} \cap C_{i j}\right), \max \left(L_{i} \cap C_{i j}\right)\right] \mid L_{i} \cap C_{i j} \neq \varnothing\right\}, \quad b_{i} \in A_{i} \cap I_{i},
$$

and for every $a \in A_{i} \cap I_{i}, a \subseteq b_{i}$. For every $i$ we now define a function

$$
g_{i}: L \cup\{-\infty, \infty\} \rightarrow L \cup\{-\infty, \infty\}: g_{i} \uparrow\left(L \cup\{-\infty, \infty\}-\bigcup_{j<\alpha_{i}} C_{i j}\right)=\mathrm{Id} ;
$$

for every $j<\alpha_{i}$, if $L_{i} \cap C_{i j}=\varnothing$, let $c_{i j} \in C_{i j}$ and for every $c \in C_{i j}$ let $g_{i}(c)=c_{i j}$; and for every $j<\alpha_{i}$, if $C_{i j} \cap L_{i} \neq \varnothing$, let $c_{i j}=\min \left(C_{i j} \cap L\right)$ and $d_{i j}=$ $\max \left(C_{i j} \cap L\right)$, let $g \uparrow\left[c_{i j}, d_{i j}\right]=\mathrm{Id}, g_{i}(c)=c_{i j}$ for every $c_{i j}>c \in C_{i j}$, and $g_{i}(c)=$ $d_{i j}$ for every $d_{i j}<c \in C_{i j}$.

Let $a_{i}=-b_{i}$. We define an endomorphism $h_{i}$ of $B(L) \uparrow a_{i}: h_{i}$ is the unique endomorphism such that, for every $\left.(c, d] \in B(L) \uparrow a_{i}, h_{i}((c, d])=\left(g_{i}(c), g_{i}(d)\right)\right]$. 
It is easy to see that $h_{i}$ is well defined and that $\left\{\left\langle A_{i}, a_{i}, h_{i}\right\rangle \mid i \in \omega\right\}$ satisfies the requirements of $P_{1}$. Q.E.D.

An uncountable subset of $\mathbf{R}$ that intersects every measure zero set in a countable set is called a Sierpinski set. Recall that $\mathrm{CH}$ implies the existence of a Sierpinski set.

A c.c.c. linear ordering which does not contain an uncountable subset order isomorphic to a subset of $\mathbf{R}$ is called a Suslin ordering.

THEOREM 6.7. (a) If $L$ is a Suslin ordering, then $L$ is thin.

(b) A Sierpinski set is thin.

(c) MA implies that every subset of $\mathbf{R}$ of cardinality less than $2^{\boldsymbol{N}_{0}}$ is thin, and that there is a thin subset of $\mathbf{R}$ of cardinality $2^{\boldsymbol{N}_{0}}$.

Proof. (a) Let $L$ be a Suslin ordering. Let $G=\left\{G_{i} \mid i \in \omega\right\}$ be an OIT for $L$, where $G_{i}=\left\{G_{i j} \mid j<\alpha_{i}\right\}$. Since $L$ is c.c.c., w.l.o.g. for every $i \in \omega, \alpha_{i}=\omega$.

Let us define the following equivalence relation on $L: x \sim y$, if for every $i, j$, $x \in G_{i j}$, iff $y \in G_{i j}$, and $x<G_{i j}$ iff $y<G_{i j}$. Clearly a set that intersects every equivalence class in at most one element is embeddable in $\mathbf{R}$, and hence the number of equivalence classes is at most $\boldsymbol{\aleph}_{0}$. Let $\left\{C_{i} \mid i \in \omega\right\}$ be an enumeration of all equivalence classes. Clearly each $C_{i}$ is $G$-small $\cup_{i \in \omega} C_{i}=L$, so $L$ is $\sigma-G$-small.

(b) Let $L$ be a Sierpinski set, and let $G$ be an OIT for $L$. It is easy to see that for every interval $[a, b]$ of $\mathbf{R}$ and every $\varepsilon>0$, there is a $G$-small closed set $F$ such that $\mu([a, b]-F)<\varepsilon$. Let $F_{i}$ be such a set for the interval $[-i, i]$ and $\varepsilon=1 / i$. Clearly $\mu\left(\mathbf{R}-\cup_{i \in \omega} F_{i}\right)=0$, and hence $\left|L-\bigcup_{i \in \omega} F_{i}\right| \leqslant \boldsymbol{\aleph}_{0}$. Let $\left\{a_{i} \mid i \in \omega\right\}=L-$ $\cup_{i \in \omega} F_{i}$, and let $C_{i}=\left(F_{i} \cap L\right) \cup\left\{a_{i}\right\}$; then $C_{i}$ is $G$-small, and $\cup_{i \in \omega} C_{i}=L$. This proves (b).

(c) Suppose MA holds. Let $L \subseteq R$ and $|L|<2^{\aleph_{0}}$, and let $G=\left\{G_{i} \mid i \in \omega\right\}$ be an OIT in $\mathbf{R}$. We shall show that there is a family $\left\{F_{i} \mid i \in \omega\right\}$ of $G$-small closed sets such that: $\mu\left(\mathbf{R}-\cup_{i \in \omega} F_{i}\right)=0$, and $L \subseteq \cup F_{i}$.

Let $G_{i}=\left\{G_{i j} \mid j \in \omega\right\}, i \in \omega$, and let $P_{\omega}(A) \stackrel{\text { def }}{=}\left\{B \subseteq A|| B \mid<\boldsymbol{\aleph}_{0}\right\}$. We define a forcing notion $\langle P, \leqslant\rangle$. Every $p \in P$ has the following form: $\left\{\left\langle\sigma_{i}^{p}, F_{i}^{p}\right\rangle \mid i \in \omega\right\}$ where: (1) for every $i \in \omega: F_{i}^{p}$ is a finite union of closed intervals and rays with rational endpoints, $\mu\left([-i, i]-F_{i}^{p}\right)<1 / i$, and $\sigma_{i}^{p} \in P_{\omega}\left(L \cap F_{i}^{p}\right)$; and (2) $\left\{i \mid F_{i}^{p} \neq\right.$ $\mathbf{R}\}$ is finite. $p \leqslant q$ if for every $i \sigma_{i}^{p} \subseteq \sigma_{i}^{q}$ and $F_{i}^{q} \subseteq F_{i}^{p}$.

It is easy to see that $P$ is c.c.c. For every $i, j \in \omega$ let $D_{i j}=\left\{p \mid\left\{k \mid F_{i}^{p} \cap G_{j k} \neq \varnothing\right\}\right.$ is finite $\}$; clearly $D_{i j}$ is dense in $P$. For every $a \in L$ let $D_{a}=\left\{p \mid a \in \cup_{i \in \omega} \sigma_{i}^{p}\right\}$; clearly $D_{a}$ is dense in $P$. Let $U$ be a filter in $P$ which intersects all the $D_{a}$ 's and all the $D_{i j}$ 's, and let $F_{i}=\bigcap_{p \in U} F_{i}^{p}$; then $\left\{F_{i} \mid i \in \omega\right\}$ is as required. This proves the first part of (c).

We now prove the second part of (c). Suppose MA holds, and let $\left\{G^{\alpha} \mid \alpha<2^{\boldsymbol{\aleph}_{0}}\right\}$ be an enumeration of all OIT's in $\mathbf{R}$. We define by induction on $\alpha$ a family $\left\{F_{i}^{\alpha} \mid i \in \omega\right\}$ of closed $G$-small sets and $a_{\alpha} \in \mathbf{R}$ such that: (1) $\mu\left(\mathbf{R}-\cup_{i \in \omega} F_{i}\right)=0$, and (2) $a_{\alpha} \in \bigcap_{\beta \leqslant \alpha} F_{i}^{\beta}-\left\{a_{\beta} \mid \beta<\alpha\right\}$.

Suppose $a_{\beta},\left\{F_{i}^{\beta} \mid i \in \omega\right\}$ has been defined for every $\beta<\alpha$. By the first part of (c), there is a family $\left\{F_{i}^{\alpha} \mid i \in \omega\right\}$ of closed $G^{\alpha}$-small sets containing $\left\{a_{\beta} \mid \beta<\alpha\right\}$, and 
such that $\mu\left(\mathbf{R}-\cup_{i \in \omega} F_{i}^{\alpha}\right)=0$. By MA $\cap_{\beta \leqslant \alpha}\left(\cup_{i \in \omega} F_{i}^{\beta}\right)-\left\{a_{\beta} \mid \beta<\alpha\right\} \neq \varnothing$; let $a_{\alpha}$ belong to this set. It is easy to see that $\left\{a_{\alpha} \mid \alpha<2^{\boldsymbol{N}_{0}}\right\}$ is thin. Q.E.D.

We now make some observations about the nonretractiveness of some free products.

Observation 6.8. (a) Let $B$ be an atomless BA such that: (1) $B-\{1\}$ is the union of countably many proper ideals (i.e. $B$ is embeddable in $P(\omega)$ ): (2) If $\left\{A_{i} \mid i \in \omega\right\}$ is an increasing sequence of subalgebras whose union is $B$, then there is $i \in \omega$ and $b \in B-\{0\}$ such that $A_{i} \uparrow b$ is dense in $B \nmid b$. Then $B$ does not have property $\mathrm{P}_{2}$.

In particular SC BA's do not have property $\mathrm{P}_{2}$.

(b) Let $A \subseteq B_{1}, B_{2}$ be uncountable BA's; then $B_{1} * B_{2}$ contains a noncountably generated ideal.

It thus follows that if $B_{1}$ is uncountable and $B$ is infinite, then $B_{1} * B_{1} * B$ is not retractive.

(c) If $B \subseteq B(\mathbf{R})$ is uncountable then $B * B$ is not retractive.

Proof. (a) Let $\left\{I_{i} \mid i \in \omega\right\}$ be a sequence of dense ideals whose union is $B-\{1\}$; let $\left\{A_{j} \mid j \in \omega\right\}$ be an increasing sequence of subalgebras whose union is $B$. We show that there are no $a_{i j}$ 's as required in $\mathrm{P}_{2}$. Let $j \in \omega$ and $b \in B-\{0\}$ be such that $A_{j} \mid b$ is dense in $B \nmid b$. Let $i \in \omega$ be such that $-b \in I_{i}$. Clearly there is no $a_{i j}$ as required in $\mathrm{P}_{2}$.

(b) Let $a \rightarrow \tilde{a}$ be an isomorphism between two copies $A$ and $\tilde{A}$ of $A$. Let $A \subseteq B$ and $\tilde{A} \subseteq \tilde{B}$; we show that ideal $I$ of $B * \tilde{B}$ generated by $C \stackrel{\text { def }}{=}\{a \cap-\tilde{a} \mid a \in A\}$ is not countably generated. If it were countably generated, then there had been a countable subset $C_{0}$ of $C$ generating $I$, but this is impossible, since for every $a \in A$ $a \cap-\tilde{a}$ does not belong to the ideal generated by $C-\{a \cap-\tilde{a}\}$.

(c) We shall prove a little bit more than what was stated in (c). A linear ordering $<$ of a subset $C$ of a BA $B$ is called a pseudo-order of $C$ relative to $B$, if for every $c_{1}, \ldots, c_{r}<c<d_{1}, \ldots, d_{s}$ in $C \cap_{i=1}^{n} d_{i} \not c \nsubseteq \cup_{i=1}^{r} c_{i}$. In such a case we shall call $C$ a pseudo-chain. Let $<_{i}$ be a pseudo-order of a subset $C_{i}$ of a BA $B_{i}, i=1,2$. We say that $C_{1}$ is pseudo-isomorphic to $C_{2}$ if $\left\langle C_{1},<_{1}\right\rangle \cong\left\langle C_{2},<_{2}\right\rangle$.

We prove the following claims.

Claim 1. If $B \subseteq B(\mathbf{R})$ is uncountable, then $B$ contains an uncountable pseudo-chain.

Claim 2. Let $C_{i} \subseteq B_{i}, i=1,2$, be pseudo-isomorphic pseudo-chains, and suppose that the subalgebra of $B_{i}$ generated by $C_{i}$ is embeddable in $P(\omega), i=1,2$. Then $B_{1} * B_{2}$ is not retractive.

Clearly (c) follows from Claims 1 and 2.

Claim 1 follows easily from the fact that $\mathbf{R}$ is separable. We prove Claim 2 . Let $<$ be a pseudo-order of $C_{1}$ relative to $B_{1}$, and $<_{2}$ be a pseudo-order of $C_{2}$. Let $c \rightarrow \tilde{c}$ be an isomorphism between $\left\langle C_{1},<\right\rangle$ and $\left\langle C_{2},<_{2}\right\rangle$. Let $I$ be the ideal in $B_{1} * B_{2}$ generated by $D \stackrel{\text { def }}{=}\left\{c \cap-\tilde{d} \mid c, d \in C_{1}\right.$ and $\left.c<d\right\}$. Our goal is to show that there is no subalgebra $A$ of $B_{1} * B_{2}$ such that for every $b \in B_{1} * B_{2}|A \cap b / I|=1$.

Let $E=\left\{c \cap-\tilde{c} \mid c \in C_{1}\right\}$, we check that $E / I$ is a set of pairwise disjoint nonzero elements in $B_{1} * B_{2} / I$. Let $c, d \in C_{1}$ and $c<d$; then $(c \cap-\tilde{c}) \cap(d \cap$ $-\tilde{d})=(c \cap d) \cap-(\tilde{c} \cup \tilde{d}) \subseteq c \cap-\tilde{d} \in I$. Hence the elements of $E$ are pairwise disjoint modulo $I$. 
Let $c \in C_{1}$ and suppose by contradiction that $c \cap-\tilde{c} \subseteq \cup_{i=1}^{m}\left(c_{i} \cap-\tilde{d}_{i}\right)$ where $c_{i}<d_{i} \in C_{1}$. W.1.o.g., for every $1 \leqslant i \leqslant k, c_{i}<c$ and, for every $k<i \leqslant m, c \leqslant c_{i}$. Let $e=\left(c-\cup_{i=1}^{k} c_{i}\right) \cap\left(\cap_{i=k+1}^{m} \tilde{d}_{i}-\tilde{c}\right)$. Clearly $0 \neq e \subseteq c \cap-\tilde{c}$ and for every $1 \leqslant i \leqslant m$, e $\cap\left(c_{i} \cap-\tilde{d}_{i}\right)=0$. Hence $c \cap-\tilde{c} \not \cup_{i=1}^{m}\left(c_{i} \cap-d_{i}\right)$.

Suppose by contradiction that there is a subalgebra $A \subseteq B_{1} * B_{2}$ such that for every $b \in B_{1} * B_{2}|A \cap b / I|=1$. For every $c \in C_{1}$ let $a_{c} \in A \cap(c \cap-\tilde{c}) / I$. $\left\{a_{c} \mid c \in C_{1}\right\}$ has to be a set of pairwise disjoint nonzero elements in $B_{1} * B_{2}$. Let $B_{i}^{\prime}$ be the subalgebra of $B_{i}$ generated by $C_{i}, i=1,2$. For every $c \in C_{1}$ there are $d_{c}^{1}, d_{c}^{2} \in I$ such that $a_{c}=\left((c \cap-\tilde{c})-d_{c}^{1}\right) \cup d_{c}^{2}$. There is $d_{c} \in D$ such that $d_{c}^{1} \subseteq d_{c}$, so $a_{c} \supseteq(c \cap-\tilde{c})-d_{c}$; since $c \cap-\tilde{c} / I \neq 0,(c \cap-\tilde{c})-d_{c} \neq 0$. Hence $\{(c \cap$ $\left.-\tilde{c})-d_{c} \mid c \in C_{1}\right\}$ is a subset of $B_{1}^{\prime} * B_{2}^{\prime}$ consisting of pairwise disjoint nonzero elements. This is impossible since $B_{1}^{\prime} * B_{2}^{\prime}$ is embeddable in $P(\omega)$ and thus it is c.c.c. Q.E.D.

REMARKs. (a) It follows from a result of Nyikos that MA $+\neg \mathrm{CH} \vDash$. If $B * B$ is retractive, then $B$ is countable.

(b) E. van Douwen has proved that if $\langle S,<\rangle$ is a Suslin ordering then $B(S) * B(S)$ is not retractive.

(c) Question 37 in [DMR] remains open; our claim quoted in note 12 there had an error. For more information about retractiveness see [DMR].

Let us state some open questions.

(1) Does ZFC imply that there is a retractive BA not embeddable in an interval algebra?

(2) Is it consistent with ZFC that $\mathbf{R}$ does not contain an uncountable thin set? ${ }^{2}$

(3) Is it consistent with ZFC that there are uncountable BA's $B_{1}$ and $B_{2}$ such that $B_{1} * B_{2}$ is retractive? Does the above follow from ZFC? What is the answer if we require in addition that $B_{1}=B_{2}$, or that $B_{1}, B_{2}$ are embeddable in $P(\omega)$ or both? What is the answer when we require that $B_{1}, B_{2}$ are interval algebras or embeddable in interval algebras or embeddable in $B(L)$, where $L=\mathbf{R}$, or $L$ is a Suslin ordering?

(4) Is it consistent with, or does it follow from ZFC that every subalgebra of a retractive $\mathrm{BA}$ is retractive?

\section{REFERENCES}

[B1] R. Bonnet, On very strongly rigid Boolean algebras and continuum discrete set condition on Boolean algebras. I, II, Algebra Universalis (submitted).

[B2] _ On very strongly rigid Boolean algebras and continuum discrete set condition on Boolean algebras. III, J. Symbolic Logic (submitted).

[Ba1] J. Baumgartner, Private communications.

[Ba2] __ Chains and antichains in Boolean algebras, preprint. Antichains in Boolean algebras, preprint.

[BaK] J. Baumgartner and R. Komjáth, Boolean algebras in which every chain and antichain is countable (submitted).

[BeN] E. Berny and P. Nyikos, Length width and breadth of Boolean algebras, Notices Amer. Math. Soc. 24 (1977); Abstract 742-06-11.

[D1] E. van Douwen, Ph. D. dissertation, Free University, Amsterdam, 1975.

${ }^{2}$ Added in proof. A. Miller answered this question positively. 
[D2] , Simultaneous linear extension of continuous functions, General Topology Appl. 5 (1975), 297-319.

[DMR] E. van Douwen, J. D. Monk and M. Rubin, Some questions about Boolean algebras, Algebra Universalis 11 (1980), 220-243.

[G] F. Galvin, Private communications.

[Mc] R. McKenzie, Private communications.

[MM] M. Magidor and J. Malitz, Compact extensions of $L(Q)$. Part la, Ann. Math. Logic 11 (1977), 217-261.

[R] B. Rotman, Boolean algebras with ordered bases, Fund. Math. 75 (1972), 187-197.

[Ru] M. Rubin, Some results in Boolean algebras, Notices Amer. Math. Soc. 24 (1977).

[S1] S. Shelah, Boolean algebras with few endomorphisms, Proc. Amer. Math. Soc. 74 (1979), 135- 142.

[S2] __ An uncountable construction, Israel J. Math. (to appear).

[W] M. Weese, Decidability of the theory of Boolean algebras with cardinality quantifiers, Bull. Acad. Polon. Sci. Sér. Sci. Math. 25 (1977), 93-97.

Department of Mathematics, Ben Gurion University of the Negev, P.O.B. 653, Beer Sheva 84120, ISRAEL 